\title{
Fully nonlinear simulations of unidirectional extreme waves provoked by strong depth transitions: the effect of slope
}

\author{
Yaokun Zheng ${ }^{1}$, Zhiliang Lin ${ }^{1, *}$ Yan $\mathrm{Li}^{2}$, T.A.A. Adcock ${ }^{2}$, Ye $\mathrm{Li}^{1}$, T.S. van den Bremer ${ }^{2}$ \\ 1 State Key Laboratory of Ocean Engineering, School of Naval Architecture, Ocean and Civil Engineering, \\ Shanghai Jiao Tong University, 200240, China \\ 2 Department of Engineering Science, University of Oxford, Parks Road, Oxford OX1 3PJ, UK
}

April 10, 2020

\begin{abstract}
Recent experimental and numerical studies of surface gravity waves propagating over a sloping bottom have shown an increase in the probability of extreme waves can be triggered by depth variations in sufficiently shallow waters. This phenomenon is studied here by means of a Boundary Element Method with fast multipole acceleration to solve the fully nonlinear water wave equations. We focus on the case of a random, unidirectional wave field with prescribed statistical properties propagating over a submerged slope, and consider different depth variations, including a step. Validation is provided by comparing with experiments by Trulsen, Zeng \& Gramstad (Phys. Fluids, 24, 097101 (2012)). Strongly non-Gaussian statistics are observed in a region localized near the depth transition, beyond which the statistics settle rapidly to the steady statistical state of finitedepth random wave fields. Using a harmonic separation technique, we show that the second-order terms are responsible for the change in the statistical properties near the depth transition. We explore in detail the effects of peak frequency, significant wave height, the inclination of the slope, and the depth of the shallower water side on the kurtosis, skewness and the excess probability of the crest height, including their spatial distributions.
\end{abstract}

\section{Introduction}

Over the past decades, different physical mechanisms have been proposed to explain the occurrence of extreme waves on the surface of the ocean $[18,8,27,1]$. Several extremely large waves have been recorded in the field, such as the Draupner [15], Andrea [23] and Killard [11] waves. Of the possible mechanisms, two general theories prevail in the open ocean and in absence of specific environmental forcing or wave-current interaction: linear random dispersive focusing [21] enhanced by weak bound-wave nonlinearity on the one hand and the modulational or Benjamin-Feir instability of waves trains in deep water on the other. The latter applies most strongly to sea states that are narrow-banded in both frequency and direction and sufficiently deep (typically, $k d \gg 1.36$, where $k$ is the wavenumber and $d$ the water depth) $[16,34,28,9]$.

Recently, the propagation of waves over strong depth transitions has been recognized an additional mechanism that can give rise to extreme waves, which we will investigate further in this paper. Trulsen et al. [31] (their Fig. 1 in particular) provides a thorough yet concise recent review of the experimental and numerical studies examining this phenomenon, as well as the different effective water depths these studies consider.

Experimentally, Trulsen et al. [32] (TZG12, hereinafter) found that there can be a local maximum of the kurtosis and skewness of the surface elevation close to the shallower side of a (1:20) underwater slope and a local maximum of the probability of large wave envelopes at the same location. In the large-scale experiments they analyze [6], long-crested waves propagate normally

*Corresponding author linzhiliang@sjtu.edu.cn 
incident to and over a sloping bottom from a deeper to a shallower domain. Similar experiments were conducted and analyzed by Zhang et al. [37] (ZBKCH19, hereinafter). The sudden peak in kurtosis and skewness has also been observed in the laboratory when waves propagate over a step Bolles et al. [4] (BSM19, hereinafter) and on top of a shoal by Trulsen et al. [31] (TRJR20, hereinafter).

Numerically, a number of authors have employed reduced-form equations to examine a possible peak in kurtosis and skewness. Sergeeva et al. [30] (SPT11, hereinafter) employed a Korteweg-de Vries (KdV) equation for variable depth, which they found did predict a local maximum (see also [24]). Examining the problem using a nonlinear Schrödinger equation (NLS) for a sloping bottom, Zeng \& Trulsen [36] (ZT12, hereinafter), in contrast, did not find the sharp peaks, although they were able to study the transition between the two statistical equilibria on both sides of the slope in more detail. Crucially, they examined much deeper water depths. The Boussinesq equations, in turn, could predict the sharp peaks, as shown by Gramstad et al. [13] (GZTP13, hereinafter) (see also [14] and [37]). Good agreement with the experimental results of TZG12 was also found by Ducrozet \& Gouin [7] (DG17, hereinafter), who used fully nonlinear simulations of the water wave equations using a higher-order spectral method. Similarly good agreement was obtained by Viotti \& Dias [33] (VD14, hereinafter), who also used fully nonlinear simulations of the water wave equations, but only made an explicit comparison to one of the three cases of TZG12. Tab. 1 summarizes the findings in the literature for waves propagating over a depth transition. The final column in Tab. 1 indicates whether peaks in both kurtosis and skewness are clearly identifiable, which we judge not to be true for case 1 of TZG12 $\left(k_{1} d_{1}=1.6, k_{2} d_{2}=0.99\right)$. Peaks in skewness and kurtosis are only predicted and observed for sufficiently shallow depths (see also the review in TRJR20).

Table 1: Summary of findings in the literature for waves propagating over a depth transition. The authors are denoted as SPT11 (Sergeeva et al. [30]), TZG12 (Trulsen et al. [32]), ZT12 (Zeng \& Trulsen [36]), GZTP13 (Gramstad et al. [13]), VD14 (Viotti \& Dias [33]), DG17 (Ducrozet \& Gouin [7]), BSM19 (Bolles et al. [4]), ZBKCH19 (Zhang et al. [37]) and TRJR20 (Trulsen et al. [31]). We estimate the slope length in VD14 as $3 \delta_{1}$ with $\delta_{1}$ their depth transition thickness. The deepest case in TZG12 and GZTP13 has a small peak in skewness but no detectable peak in kurtosis.

\begin{tabular}{|c|c|c|c|c|c|}
\hline Authors & $\begin{array}{c}\text { Deeper depth } \\
k_{1} d_{1}\end{array}$ & $\begin{array}{l}\text { Shallower depth } \\
\qquad k_{2} d_{2}\end{array}$ & $\begin{array}{l}\text { Slope } \\
-\partial d / \partial x\end{array}$ & Method & Peak? \\
\hline SPT11 & 0.44 & 0.3 & 0.03 & $\mathrm{KdV}$ for sloping bottom & Yes \\
\hline TZG12 & 1.6 & 0.99 & 0.05 & Experiments & No \\
\hline " & 1.1 & 0.70 & $"$ & " & Yes \\
\hline$"$ & 0.81 & 0.54 & $"$ & $"$ & Yes \\
\hline $\mathrm{ZT} 12$ & 10 & $1.2-4.0$ & $0.03-0.045$ & NLS for sloping bottom & No \\
\hline GZTP13 & 1.6 & 0.99 & 0.05 & Boussinesq & No \\
\hline " & 1.1 & 0.70 & $"$ & $"$ & Yes \\
\hline$"$ & 0.81 & 0.54 & $"$ & $"$ & Yes \\
\hline VD14 & 1.8 & $0.78-1.03$ & $0.13-0.21$ & Fully nonlinear & Yes \\
\hline$\overline{D G} 17$ & 1.6 & 0.99 & 0.05 & Fully nonlinear & No \\
\hline$"$ & 1.1 & 0.70 & $"$ & " & Yes \\
\hline$"$ & 0.81 & 0.54 & $"$ & $"$ & Yes \\
\hline BSM19 & 2.1 & 0.76 & $\infty$ & Experiments & Yes \\
\hline ZBKCH19 & 1.19 & 0.50 & 0.05 & Experiments & Yes \\
\hline " & $"$ & $"$ & $"$ & Boussinesq & Yes \\
\hline$"$ & $"$ & $"$ & $"$ & Fully nonlinear & Yes \\
\hline TRJR20 & $1.8-3.8$ & $0.54-1.3$ & 0.26 & Experiments & Yes \\
\hline " & $4.3-4.9$ & $1.4-1.6$ & " & Experiments & No \\
\hline
\end{tabular}

Moving from uni-directional to directional wave fields, Janssen \& Herbers [17] found that refraction in a focal zone could lead to an increase in extreme wave occurrence, whereas DG17 found 


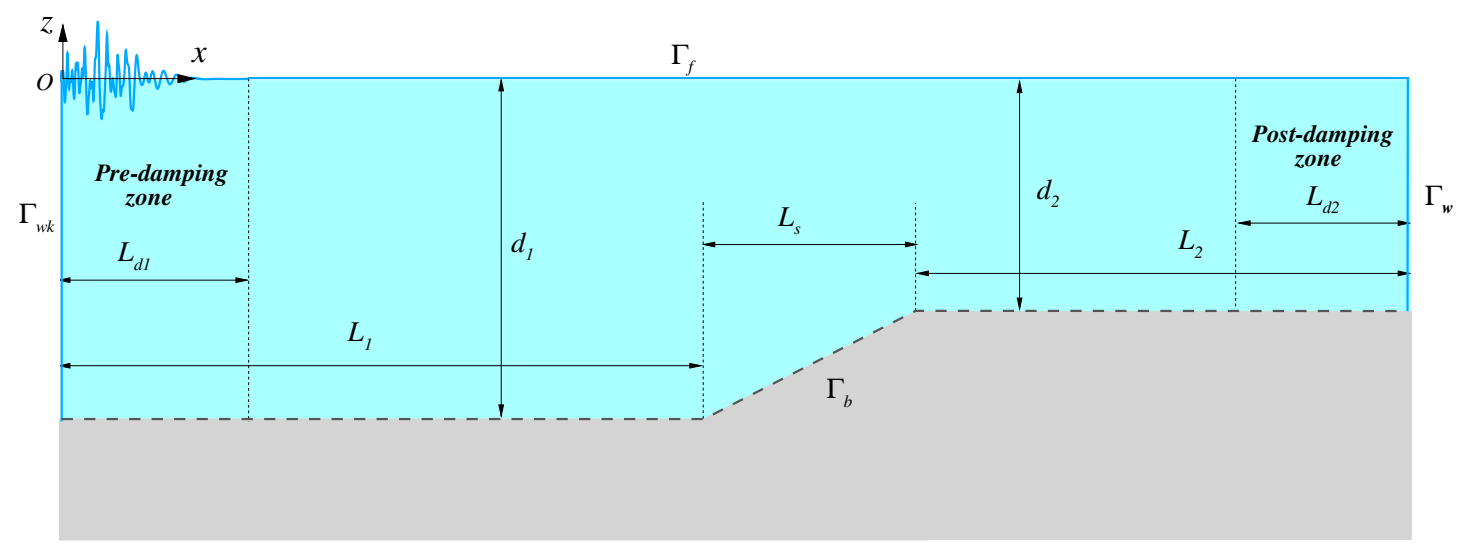

Figure 1: Side view of the two-dimensional numerical wave tank.

that extreme wave occurrence could be reduced for directional spread sea states.

Although previous experimental and numerical studies have provided quantitative insight into the magnitude of the peaks in kurtosis and skewness and their dependence on the water depths for which they occur, the physical mechanism for the relationship between extreme wave generation and strong depth transitions is not well understood, and a theoretical model does not exist. VD14 [33] have interpreted the behaviour as a transition between two different statistical equilibria with the sharp peaks a result of out-of-equilibrium dynamics. Through bi-spectral analysis, ZBKCH19 [37] have demonstrated that the sharp peaks are likely a result of second-order (in amplitude) effects, which we investigate further in this paper. A similar conclusion has previously been reached by GTZP13 [13] using numerical simulations of the Boussinesq equations.

In this paper, we use fully nonlinear simulations of the water wave equations using a Numerical Wave Tank (NWT) developed by [19] based on the Boundary Element Method to study the physical mechanism behind the relationship between extreme wave generation and strong depth transitions. We have increased the efficiency of this NWT using the Fast Multipole Method to make it suitable for random simulations. We take as a starting point the experimental work of Trulsen, Zeng \& Gramstad [32] (TZG12), who considered the propagation of three sets of long-crested irregular wave normally incident onto and over a non-uniform bathymetry consisting of a 1:20 slope from a constant water depth of $0.6 \mathrm{~m}$ to a shallower constant water depth of $0.3 \mathrm{~m}$. We begin by comparing our results to the three cases in TZG12. Taking case 2 in TZG12 as a base case, we then vary the significant wave height, length of the slope and depth of the shallower side. Compared to GZTP13, who also varied significant wave height, length of the slope and depth of the shallower side using the Boussinesq equations, we study a greater range of slopes, including a step or infinitely steep slope. Furthermore, we study both the magnitude of the peaks in kurtosis and skewness and the location at which they occur. Finally, we use harmonic separation [2] to examine the order in a hypothetical Stokes-like expansion at which the mechanism connecting strong depth transitions to extreme waves arises.

This paper is laid out as follows. First, $\S 2$ introduces the governing equations and the numerical model, followed by the model setup and parameter choices in $\S 3$ and a convergence study in Appendix A. Results are discussed in $\S 4$, followed by conclusions in $\S 5$.

\section{Governing equations and numerical model}

In this paper, we use potential flow theory to model wave propagation. The numerical model employed for the simulations is a fully nonlinear numerical wave tank (NWT) based on the Boundary Element Method developed by [19], which we enhance using fast multipole acceleration. We consider the propagation of a wave train over a sloping bottom from a deeper water depth $d_{1}$ to a shallower water depth $d_{2}$, as shown in Fig. 1. A Cartesian coordinate system is used with its origin located at the still water level on the deeper end of the tank. The NWT has damping zones at both ends, and their lengths are set as $L_{d 1}$ and $L_{d 2}$, respectively. The length between the input boundary and the beginning of the slope is $L_{1}$, the horizontal length of the slope is $L_{s}$, and the 
length between the end of the slope and the end of the wave tank is $L_{2}$. The values of all these parameters will be set in the next section.

\subsection{Governing equations}

The velocity potential $\phi(x, z, t)$ and free surface elevation $\eta(x, t)$ of the fluid domain $\Omega(t)$ are used to describe the inviscid, irrotational flow, neglecting surface tension. The velocity field is defined as $\boldsymbol{u}=\boldsymbol{\nabla} \phi$, and the Laplace equation for the potential flow field can be obtained as

$$
\nabla^{2} \phi=0 \quad \text { in } \Omega(t)
$$

from the continuity equation in the fluid domain with $\boldsymbol{\nabla}=(\partial / \partial x, \partial / \partial z)$.

\subsection{Boundary and initial conditions}

The incoming irregular wave field is prescribed in terms of a superposition of $M$ linear harmonic wave components; the surface elevation $\eta$ and the velocity potential $\phi$ are given as

$$
\begin{aligned}
\eta(x, t) & =\sum_{i=1}^{M} a_{i} \cos \left(k_{i} x-\omega_{i} t+\theta_{i}\right), \\
\phi(x, z, t) & =\sum_{i=1}^{M} \frac{g a_{i}}{\omega_{i}} \frac{\cosh \left(k_{i}\left(z+d_{1}\right)\right)}{\cosh \left(k_{i} d_{1}\right)} \sin \left(k_{i} x-\omega_{i} t+\theta_{i}\right),
\end{aligned}
$$

where $a_{i}, \omega_{i}, k_{i}$, and $\theta_{i}$ are, respectively, the amplitude, angular frequency, wavenumber, and phase of the $i$ th wave component. The wavenumber $k_{i}$ is obtained using the linear dispersion relation $\omega_{i}^{2}=g k_{i} \tanh \left(k_{i} d_{1}\right)$, and $d_{1}$ is the depth of the deeper side. Hence, the input boundary condition is defined as

$$
\frac{\partial \phi(x, z, t)}{\partial n}=\frac{\partial \phi}{\partial x} n_{x}=-\sum_{i=1}^{M} \frac{g a_{i} k_{i}}{\omega_{i}} \frac{\cosh \left(k_{i}\left(z+d_{1}\right)\right)}{\cosh \left(k_{i} d_{1}\right)} \cos \left(k_{i} x-\omega_{i} t+\theta_{i}\right) \quad \text { on } \Gamma_{w k},
$$

as $n_{x}=-1$. For numerical stability, we apply a modulation function $\tilde{f}(t)$ when the simulation starts to prevent impulse-like behavior of wavemaker; the modulation function can be written as

$$
\tilde{f}(t)= \begin{cases}\frac{1}{2}[1-\cos (\pi t / \tilde{T})] & t \leq \tilde{T} \\ 1 & t>\tilde{T}\end{cases}
$$

where $\tilde{T}$ is the modulation duration. We set $\tilde{T}=2 T_{p}$ for all simulated cases with $T_{p}$ the peak period of the irregular waves. At the other end of the wave tank and at the bottom of the seabed including the sloped part, the boundaries are considered to be impermeable and satisfy the no-normal-flow condition

$$
\frac{\partial \phi}{\partial n}=0 \text { on } \Gamma_{b} \text { and } \Gamma_{w}
$$

On the free surface $z=\eta(x, t)$, the fully nonlinear kinematic and dynamic boundary conditions are defined as

$$
\frac{\partial \eta}{\partial t}=-\frac{\partial \phi}{\partial x} \frac{\partial \eta}{\partial x}+\frac{\partial \phi}{\partial z}, \quad \frac{\partial \phi}{\partial t}=-g \eta-\frac{1}{2}|\nabla \phi|^{2} .
$$

Initially, at the start of the simulation, the free surface is undisturbed. For the initial boundary value problem, the initial conditions are

$$
\phi=\eta=0 \quad \text { for } \quad x \in \Gamma_{f}, t=0 .
$$

With all the boundary and initial conditions defined, the unsteady velocity potential is solved using a Boundary Element Method (BEM) with a simplified Green's function G (e.g. [29]). The numerical wave tank we thus develop is based on [19]. The boundary integral equation is

$$
c_{i} \phi_{i}=\int_{\Gamma}\left(G_{i j} \frac{\partial \phi_{j}}{\partial n}-\frac{\partial G_{i j}}{\partial n} \phi_{j}\right) \mathrm{d} \Gamma_{j},
$$


where $\phi_{i}$ is velocity potential at the source point $\left(x_{i}, z_{i}\right), c_{i}$ is the solid angle factor for the source point on the domain boundary $\Gamma$ where $\Gamma=\Gamma_{f} \cup \Gamma_{w k} \cup \Gamma_{b} \cup \Gamma_{w}, \phi_{j}$ is the velocity potential at the field point $\left(x_{j}, z_{j}\right), c_{i}$ is the solid angle factor for the source point on the domain boundary $\Gamma$, and the Green's function $G_{i j}=-\ln \left(r_{i j}\right) / 2 \pi$, where $r_{i j}$ is the distance between the source point and the field point, satisfies the Laplace equation. The domain boundary $\Gamma$ consists of the free surface boundary $\Gamma_{f}$, the wavemaker boundary $\Gamma_{w k}$, the vertical boundary at the opposite end $\Gamma_{w}$ and the bottom boundary $\Gamma_{b}$, as shown in Fig. 1.

\subsection{Mixed Eulerian and Lagragian method}

In this paper, the Mixed Eulerian-Lagrangian (MEL) method first introduced by Longuet Higgins and Cokelet [22] is employed to update the free surface elevation in time. At each time step, we solve for the boundary values in the Eulerian frame by using the Boundary Element Method. Between time steps, we update the boundary values and boundary element nodes on the free surface in Lagrangian manner. Applying the material derivative $\mathrm{D} / \mathrm{D} t=\partial / \partial t+\boldsymbol{u} \cdot \boldsymbol{\nabla}$, with $\boldsymbol{u}=\boldsymbol{\nabla} \phi$ the Eulerian velocity, the fully nonlinear free surface boundary conditions become [22]

$$
\frac{\mathrm{D} \phi}{\mathrm{D} t}=-g \eta+\frac{1}{2}|\nabla \phi|^{2}, \quad \frac{\mathrm{D} \eta}{\mathrm{D} t}=\frac{\partial \phi}{\partial z} .
$$

A classical fourth-order Runge-Kutta scheme is used as the time-marching method for updating the free surface profile and the values of the velocity potential, which has been proven sufficiently stable for fully nonlinear simulations of a numerical wave tank [19].

\subsection{Numerical damping zones}

We use damping zones at both ends of the tank to dissipate the wave energy and minimize wave reflections (see Fig. 1). A post-damping zone on the right-hand side of the tank prevents reflections of the incoming wave train (see [19]). Before the waves reach this end, waves may interact with the sloping bottom and reflect, causing re-reflection at the input boundary. The pre-damping zone on left-hand side of the wave tank is introduced to suppress re-reflection. In the post-damping zone, damping terms are added into the dynamic and kinematic free surface boundary conditions. A ramp function is employed to avoid impulse-like behaviour at the beginning of damping zone (see [19]). The free surface boundary conditions (10) in the post-damping zone then become:

$$
\frac{\mathrm{D} \phi}{\mathrm{D} t}=-g \eta+\frac{1}{2}|\nabla \phi|^{2}+\mu_{1} \frac{\partial \phi}{\partial n}, \quad \frac{\mathrm{D} \eta}{\mathrm{D} t}=\frac{\partial \phi}{\partial z}+\mu_{2} \eta
$$

with ramp function

$$
\mu_{i}= \begin{cases}\mu_{0 i}\left[1-\cos \left(\frac{\pi}{2}\left(\frac{x-x_{d 2}}{L_{d 2}}\right)\right)\right] & x>x_{d 2}, \\ 0 & x \leq x_{d 2},\end{cases}
$$

where $x_{d 2}$ is the location where the post-damping zone begins, and $L_{d 2}$ is the length of the postdamping zone. After thorough testing, the dimensional coefficients are chosen to be $\mu_{01}=2.8 \omega_{p} / k_{p}$ and $\mu_{02}=k_{p} \mu_{01}$, in which the peak wavenumber $k_{p}$ is obtained from the peak period $T_{p}$ through the linear dispersion relationship. Likewise, damping terms are added in the pre-damping zone. There, the damping terms are designed to dampen the reflected wave without influencing the incident wave:

$$
\frac{\mathrm{D} \phi}{\mathrm{D} t}=-g \eta+\frac{1}{2}|\nabla \phi|^{2}+\hat{\mu}_{1}\left(\frac{\partial \phi}{\partial n}-\frac{\partial \phi^{*}}{\partial n}\right), \quad \frac{\mathrm{D} \eta}{\mathrm{D} t}=\frac{\partial \phi}{\partial z}+\hat{\mu}_{2}\left(\eta-\eta^{*}\right),
$$

with ramp function

$$
\hat{\mu}_{i}= \begin{cases}\mu_{0 i}\left[1-\cos \left(\frac{\pi}{2}\left(\frac{x_{d 1}-x}{L_{d 1}}\right)\right)\right] & x \leq x_{d 1}, \\ 0 & x>x_{d 1},\end{cases}
$$

where $\partial \phi^{*} / \partial n$ and $\eta^{*}$ are the target velocity and surface elevation inputs, $x_{d 1}$ is the end of the pre-damping zone, and the dimensional coefficients $\mu_{01}=2.8 \omega_{p} / k_{p}$ and $\mu_{02}=k_{p} \mu_{01}$ are the same as in the post-damping zone. In this paper, the length of damping zones $L_{d i}$ is set as 6 times the peak wavelength of the incoming irregular wave field. 


\subsection{Fast multipole acceleration for BEM}

To help discover the physical mechanism for the relationship between extreme wave generation and strong depth transitions, it is important to have an efficient and accurate numerical model. Evidently, to study the long-time evolution and higher-order statistical moments of an irregular wave field in a long numerical tank, efficient and accurate numerical simulation is imperative. To do so, we choose a combination of the conventional Boundary Element Method (BEM) [5] and the Fast Multipole Method (FMM). Using the FMM, we overcome the curse of dimensionality that would be prohibitive for the large domain and high mesh density required for the problem considered here (see Yoshida [35], Nishimura [26], Liu \& Nishimura [20]). The Numerical Wave Tank (NWT) based on the Fast Multipole Boundary Element Method (FMBEM) we develop in this paper (i) reduces the computational dimension from a two-dimensional problem to a one-dimensional problem, (ii) does not have a limitation on the size of the tank and the wave height as long as wave breaking does not occur, (iii) has good convergence properties for intermediate water depths and (iv) has a high efficiency for time-domain simulations for a very large number of discretized boundary elements compared to the standard Boundary Element Method on which it is based [19]. Compared to other numerical methods, our BEM-based NWT is still relatively computationally demanding, with a single case taking approximately 4 days on a single-core processor.

With boundary conditions given and after discretization, the next step is to move the unknown variables to the left-hand side and known variables to the right-hand side, so that the equations can be written in a matrix form $A \boldsymbol{\lambda}=\boldsymbol{b}$, where the matrix $A$ and the vector $\mathbf{b}$ are known, and the vector $\boldsymbol{\lambda}$ is unknown. All the boundary values are then obtained by solving this matrix equation.

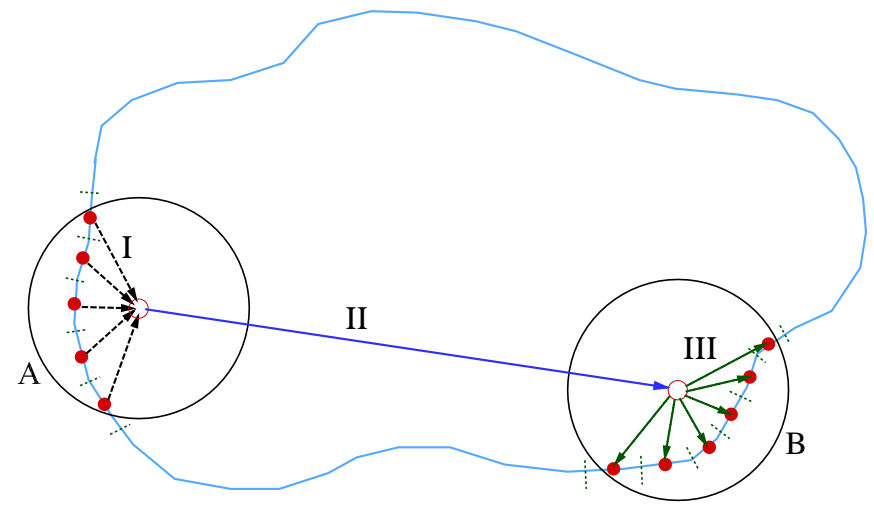

Figure 2: Demonstration of the Fast Multipole Method (FMM) and its three constituent processes: Moment Concentration (Process I), Moment Translation (Process II) and Moment Expansion (Process III).

Fast Multipole Boundary Element Methods (FMBEMs) apply iterative solvers, such as the Generalized Minimal Residual Method (GMRES), to solve the boundary integral equation and use the Fast Multipole Method (FMM) to accelerate the matrix multiplication in each iteration without forming the entire matrix. As shown in Fig. 2, we use constant boundary elements in our discretization scheme, that is, we divide the boundary into $N$ line elements and place one node on each element. Then, we divide the elements into different sets according to their spatial positions (e.g., cell A and cell B, as shown in Fig. 2). The main principle of FMM is to translate the element-to-element interactions to cell-to-cell interactions. First, the so-called moments within each cell, i.e. the integral of the Green's function kernel over the boundary of the source elements, are concentrated at the center point (Process I: Moment Concentration). Then, the moments are translated from cell to cell by upward and downward passes through the defined tree structure of the mesh (Process II: Moment Translation). Finally, the moments are distributed out from the center to the collocation points within the cell (Process III: Moment Expansion). Further details can be found in Liu and Nishimura [20]. Using the FMM, the solution time of a problem can be reduced to $O(N \log N)$. The memory requirement can also be reduced to $O(N)$ since iterative solvers do not need to store the entire matrix in the memory. 


\section{Model setup}

In this paper, we use the FMBEM to simulate irregular waves propagating over a depth transition in the form of a sloped bottom. To begin, the geometry of the computational domain is defined to correspond to the experiments of TZG12 as a benchmark. The computational domain is defined as depicted in Fig. 1, and the only difference with TZG12 is the presence of damping zones at both ends of the numerical wave tank. To ensure the damping zone at the end of the tank does not have any effect at the probe locations of TZG12, the length of the shallow side is extended relative to the experiments.

\subsection{Matrix of numerical experiments}

Tab. 2 summarizes the different cases we consider. The peak wavenumber $k_{p}$ is approximated using the linear dispersion relation $\omega_{p}^{2}=4 \pi^{2} / T_{p}^{2}=g k_{p} \tanh \left(k_{p} d\right)$, where $T_{p}$ is the peak period. In the table, $k_{p 1}$ and $d_{1}$ correspond to the deeper side, while $k_{p 2}$ and $d_{2}$ correspond to the shallower side. The characteristic amplitude $a_{c}=H_{s} /(2 \sqrt{2})$ with $H_{s}$ the significant wave height.

First, we examine the three cases considered in the experiments of TZG12, which have the same significant wave heights $H_{s}$ but three different peak periods $T_{p}$ (our cases A1-A3, corresponding to cases 1-3 in TZG12). Second, we investigate the effect of the significant wave height $H_{s}$ of the irregular waves, keeping the peak period $T_{p}$ constant (cases B1-B6). More importantly, we then consider a wide range of different slopes (cases C1-C7). To do so, we keep the depths on both the deeper and the shallower sides constant but change the length of the slope. We also consider the case when the length of the slope becomes zero, and the slope becomes a step. Finally, we consider the influence of the depth of the shallower water side on the occurrence of the extreme waves (cases D1-D3). Keeping the depth of the deeper side and the horizontal length of the slope unchanged, we vary the depth of the shallower side and thus the slope.

Table 2: Matrix of all numerical experiments $\left(L_{1}=143.41 \mathrm{~m}\right.$ and $\left.d_{1}=0.6 \mathrm{~m}\right)$.

\begin{tabular}{lccccccccc}
\hline Case & $T_{p}[\mathrm{~s}]$ & $H_{s}[\mathrm{~m}]$ & $L_{s}[\mathrm{~m}]$ & $d_{2}[\mathrm{~m}]$ & slope & $k_{p 1} d_{1}$ & $k_{p 1} a_{c 1}$ & $k_{p 2} d_{2}$ & $k_{p 2} a_{c 2}$ \\
\hline $\mathrm{A} 1$ & 1.27 & 0.06 & 6 & 0.3 & $1: 20$ & 1.61 & 0.057 & 0.99 & 0.070 \\
$\mathrm{~A} 2^{*}$ & 1.70 & 0.06 & 6 & 0.3 & $1: 20$ & 1.06 & 0.038 & 0.70 & 0.049 \\
$\mathrm{~A} 3$ & 2.12 & 0.06 & 6 & 0.3 & $1: 20$ & 0.81 & 0.028 & 0.54 & 0.038 \\
\hline $\mathrm{B} 1$ & 1.70 & 0.03 & 6 & 0.3 & $1: 20$ & 1.06 & 0.019 & 0.70 & 0.025 \\
$\mathrm{~B} 2$ & 1.70 & 0.04 & 6 & 0.3 & $1: 20$ & 1.06 & 0.025 & 0.70 & 0.033 \\
$\mathrm{~B} 3$ & 1.70 & 0.05 & 6 & 0.3 & $1: 20$ & 1.06 & 0.031 & 0.70 & 0.041 \\
$\mathrm{~B} 4{ }^{*}$ & 1.70 & 0.06 & 6 & 0.3 & $1: 20$ & 1.06 & 0.038 & 0.70 & 0.049 \\
$\mathrm{~B} 5$ & 1.70 & 0.07 & 6 & 0.3 & $1: 20$ & 1.06 & 0.044 & 0.70 & 0.058 \\
$\mathrm{~B} 6$ & 1.70 & 0.08 & 6 & 0.3 & $1: 20$ & 1.06 & 0.050 & 0.70 & 0.066 \\
\hline $\mathrm{C} 1$ & 1.70 & 0.06 & 0 & 0.3 & $\infty$ & 1.06 & 0.038 & 0.70 & 0.049 \\
$\mathrm{C} 2$ & 1.70 & 0.06 & 1.5 & 0.3 & $1: 5$ & 1.06 & 0.038 & 0.70 & 0.049 \\
$\mathrm{C} 3$ & 1.70 & 0.06 & 3 & 0.3 & $1: 10$ & 1.06 & 0.038 & 0.70 & 0.049 \\
$\mathrm{C} 4^{*}$ & 1.70 & 0.06 & 6 & 0.3 & $1: 20$ & 1.06 & 0.038 & 0.70 & 0.049 \\
$\mathrm{C} 5$ & 1.70 & 0.06 & 12 & 0.3 & $1: 40$ & 1.06 & 0.038 & 0.70 & 0.049 \\
$\mathrm{C} 6$ & 1.70 & 0.06 & 18 & 0.3 & $1: 60$ & 1.06 & 0.038 & 0.70 & 0.049 \\
$\mathrm{C} 7$ & 1.70 & 0.06 & 24 & 0.3 & $1: 80$ & 1.06 & 0.038 & 0.70 & 0.049 \\
\hline $\mathrm{D} 1 *$ & 1.70 & 0.06 & 6 & 0.3 & $1: 20$ & 1.06 & 0.038 & 0.70 & 0.049 \\
$\mathrm{D} 2$ & 1.70 & 0.06 & 6 & 0.35 & $1: 24$ & 1.06 & 0.038 & 0.76 & 0.046 \\
$\mathrm{D} 3$ & 1.70 & 0.06 & 6 & 0.4 & $1: 30$ & 1.06 & 0.038 & 0.82 & 0.044 \\
\hline
\end{tabular}

${ }^{*}$ Cases A2, B4, C4 and D1 share identical parameters.

\subsection{Wave spectrum}

The target spectrum of the incident irregular waves at the beginning of the numerical wave tank is the modified JONSWAP-type spectrum presented by Goda [12] with peak angular frequency $\omega_{p}$, 


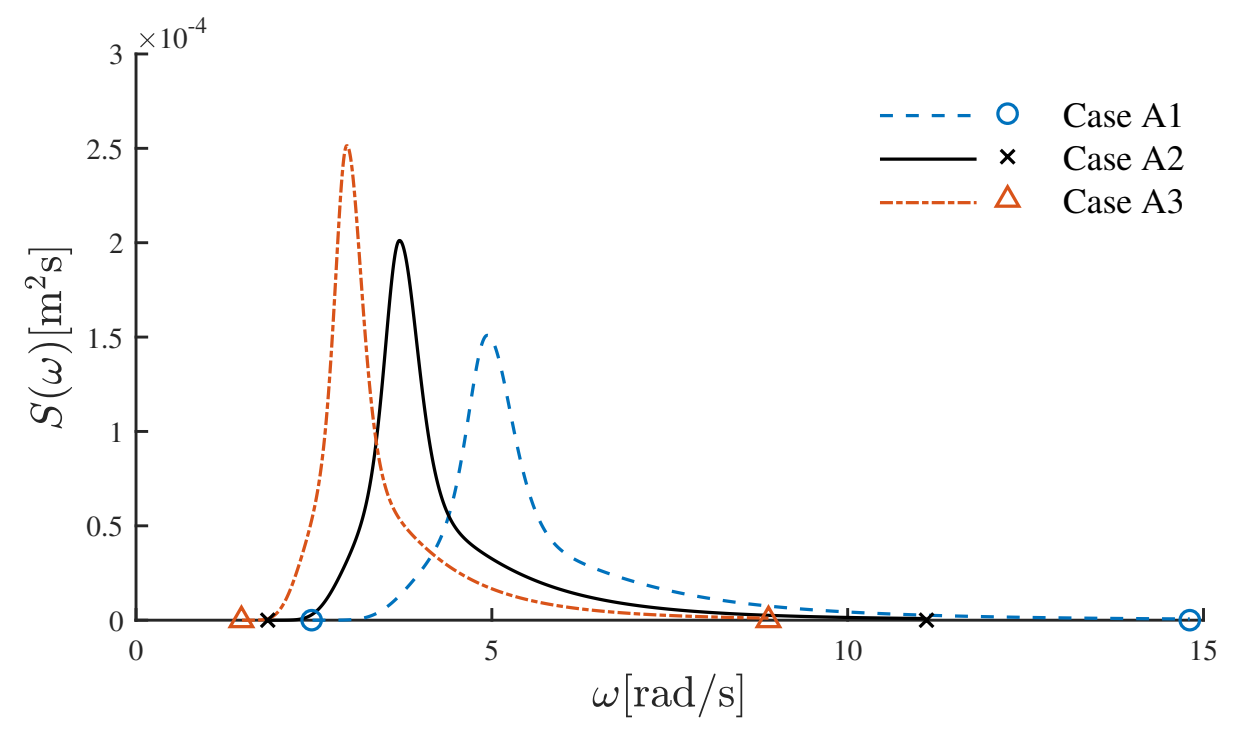

Figure 3: Three spectra corresponding to our cases A1-A3 and cases 1-3 in TZG12's experiments (see Tab. 2). The markers show the lower-bound and upper-bound cut-offs chosen for the frequency interval $\left(\left[0.5 \omega_{p}, 3 \omega_{p}\right]\right)$.

significant wave height $H_{s}$ and peak enhancement factor $\gamma$ :

$$
\begin{aligned}
& S(\omega)=\frac{2 \pi \beta H_{s}^{2} \omega_{p}^{4}}{\omega^{5}} \exp \left[-1.25\left(\frac{\omega_{p}}{\omega}\right)^{4}\right] \gamma^{\exp \left[-\frac{\left(\omega-\omega_{p}\right)^{2}}{2 \sigma^{2} \omega_{p}^{2}}\right],} \\
& \beta=\frac{0.06238}{0.230+0.0336 \gamma-\frac{1}{0.185(1.9+\gamma)}}(1.094-0.01915 \ln \gamma) .
\end{aligned}
$$

The peak enhancement factor is chosen as $\gamma=3.3$ in all the numerical cases. The spectral asymmetry parameter $\sigma$ is set as $\sigma=0.07$ when $\omega<\omega_{p}$ and $\sigma=0.09$ when $\omega>\omega_{p}$.

Fig. 3 shows the three spectra of the irregular waves corresponding to cases 1-3 in TZG12's experiments, which are also used in this paper (cases A1-A3). A finite range of frequencies $\left[\omega_{\min }, \omega_{\max }\right]$, which captures practically all of the energy, is taken for the irregular wave simulations. That is, below the lower-bound frequency $\omega_{\min }$ and beyond the upper-bound frequency $\omega_{\max }, S(\omega)$ is nearly identically zero. As shown in Fig. 3, in all these three cases we can let $\omega_{\min }=0.5 \omega_{p}$ and $\omega_{\max }=3 \omega_{p}$, and our cut-offs do not result in a significant part of the energy of the spectrum being ignored. Furthermore, the smallest wave length $\left(\lambda_{\min }\right)$ corresponding to the frequency upper bound must not be too large compared to the size of the discrete elements.

To obtain a random irregular sea state, we divide the spectrum into $M$ discrete components with the same energy. As a result of the equal distribution of energy, the frequency components are unevenly distributed, and we explain how they are calculated as follows. The amplitude of each wave component is the same, $a=\sqrt{2 E / M}$ with $E=\int_{\omega_{\min }}^{\omega_{\max }} S(\omega) \mathrm{d} \omega$, where $\omega_{\min }=0.5 \omega_{p}$ and $\omega_{\max }=3 \omega_{p}$ in the simulations. For the $i$ th wave component with frequency $\omega_{i}$, we have the identity

$$
\frac{E}{M}=\int_{\omega_{i}^{-}}^{\omega_{i}^{+}} S(\omega) \mathrm{d} \omega .
$$

We then calculate the bins $\omega_{i}^{ \pm}$starting from $\omega_{\min }$ and use the average frequency for each bin, $\omega_{i}=\left(\omega_{i}^{-}+\omega_{i}^{+}\right) / 2$. We set $M=200$ for all cases. The phase of each component $\theta_{i}$ is then assigned randomly from a uniform distribution on $[0,2 \pi)$. Defining only the phases randomly, as we have done, places an upper limit on the maximum crest elevation as the deterministic sum of all the components when they are in phase. In practise, we use sufficient components that the restriction of only random phases, a commonly made assumption, does not affect the wave statistics at the probability levels we consider, including the statistical moments discussed next.

To characterize extreme wave occurrence, we examine the third and fourth-order statistical moments of the probability density function. We will estimate the skewness (standardized thirdorder moment $\lambda_{3}$ ) and the kurtosis (standardized fourth-order moment $\lambda_{4}$ ) of the free surface 
elevation $\eta(x, t)$ at different locations $x$ from the random signals in the time domain as

$$
\lambda_{3}=\frac{\left\langle(\eta-\langle\eta\rangle)^{3}\right\rangle}{\sigma^{3}} \quad \text { and } \quad \lambda_{4}=\frac{\left\langle(\eta-\langle\eta\rangle)^{4}\right\rangle}{\sigma^{4}}
$$

where $\langle\cdot\rangle$ denotes an average over time, and $\sigma$ is the standard deviation of $\eta$ (i.e., $\sigma^{2}=\left\langle(\eta-\langle\eta\rangle)^{2}\right\rangle$ ), from which the the significant wave height can be obtained $\left(H_{s}=4 \sigma\right)$.

We recall that skewness characterizes the asymmetry of the distribution with respect to the mean, while kurtosis measures the importance of the tails and thus extremes. For a surface elevation that is a Gaussian random process, the skewness can be shown to be exactly equal to 0 and the kurtosis exactly equal to 3. Larger values of the kurtosis indicate the presence of extreme events. We can confirm from our (linear) input boundary conditions that we obtain a skewness of 0.0 and a kurtosis of 3.0 with only random phases (and $M=200$ ).

\subsection{Convergence}

In addition to the geometry of the computational domain, it is necessary to define carefully the numerical parameters for an accurate description of the evolution of the sea state of interest, such as the size of the elements (compared to smallest wavelength present). Furthermore, the duration of the simulation must be long enough to obtain stable statistical moments. To address this, a convergence study has been carried out, and details are given in Appendix A. In summary, we set the mesh density as 25 and we use $M=200$ random components. The mesh density is defined as the number of boundary elements per $\lambda_{\min }$, where $\lambda_{\min }$ is the smallest wave length and corresponds to the upper-bound cut-off of the frequency spectrum (i.e., $3 \omega_{p}$ ).

Although we have performed a convergence study for case A2 (see Appendix A), it is evident from Fig. 4 that the variance decreases by approximately $10 \%$ across the deeper side for case A1. This behaviour, which is probably indicative of lack of convergence, is not present in the other cases considered in this paper. The reason for this behaviour is that case A1 is the only modulationally unstable case $\left(k_{p 1} d_{1}=1.61>1.36\right)$, combined with its relatively large steepness $\left(k_{p 1} a_{c 1}=0.057\right.$, the largest of all cases). This places a significant demand on our code. As modulational unstable cases are not the focus of this study, we do not investigate this further herein. However, we emphasize that the results for case A1 (only) should be treated with caution.

\subsection{Harmonic extraction}

To examine the role of second-order waves, we use the so-called two-phase harmonic extraction (or phase inversion) technique ([2], see also [10, 25]). To do so, we carry out each simulation in duplicate form with the phases $\theta_{i}$ of the input signal at the input boundary condition to the second simulation shifted by $\pi$. Thus, two times series $\eta_{0}(t)$ and $\eta_{\pi}(t)$ can be obtained, and harmonic components of odd and even powers in amplitude can be separated:

$$
\begin{aligned}
\eta_{\text {odd }} & =\frac{\eta_{0}-\eta_{\pi}}{2}=\eta^{(1,1)}+\eta^{(3,1)}+\eta^{(3,3)}+\ldots, \\
\eta_{\text {even }} & =\frac{\eta_{0}+\eta_{\pi}}{2}=\eta^{(2,0)}+\eta^{(2,2)}+\eta^{(2,4)}+\ldots,
\end{aligned}
$$

where the first index in the superscript refers to the power in amplitude, while the second refers to the harmonic of the underlying Stokes expansion. To leading order therefore, $\eta_{\text {odd }}$ is dominated by the first-order (in amplitude) signal, and $\eta_{\text {even }}$ by the second-order (in amplitude) signal. Despite the approximate nature of the identity, we will refer to $\eta_{\text {odd }}$ as the linear signal and to $\eta_{\text {even }}$ as the second-order signal below.

\section{Numerical results and discussion}

For every numerical experiment in Tab. 2, we record time series of surface elevation on a closelyspaced grid in the $x$-direction. For every point in $x$, we then calculate the skewness, kurtosis and the exceedance probability of the Hilbert envelope from the time series. The Hilbert envelope is calculated as $A(t)=\sqrt{(\eta(t))^{2}+(\tilde{\eta}(t))^{2}}$, where $\tilde{\eta}(t)$ is the Hilbert transform of the time series 
of surface elevation $\eta(t)$. In this section, we will begin by comparing our simulations to the experiments of TZG12 (§4.1), and then vary the significant wave height ( $\$ 4.2)$, the length of the slope (§4.3), and the depth of the shallower side (§4.4).

\subsection{Comparison with the experiments of TZG12}

\subsubsection{Spatial profiles of the statistical moments}

In Figs. 4-6, we compare the results from our numerical simulations to the experiments of TZG12, showing variance, skewness and kurtosis. Generally, the numerical results are consistent with the experiments of TZG12, with better agreement for the normalized quantities skewness and kurtosis than for the unnormalized variance. The kurtosis starts at a value of 3.0 consistent with a Gaussian random process. The starting value of the skewness of 0.0 reflects linear generation at the wavemaker and is consistent with a linear signal that follows a symmetric Gaussian random process; the small positive values further down the tank represent second-order bound waves being established. Both numerical and experimental results show that the kurtosis (for cases A2 and A3) and skewness (for cases A1, A2 and A3) reach a local maximum at a location near the shallower edge of slope. Fig. 7 gives the exceedance probability of the Hilbert envelope at the locations where the kurtosis and skewness reach their maxima, demonstrating an increased likelihood of extreme waves compared to the Rayleigh distribution, and increasingly so for for larger periods.
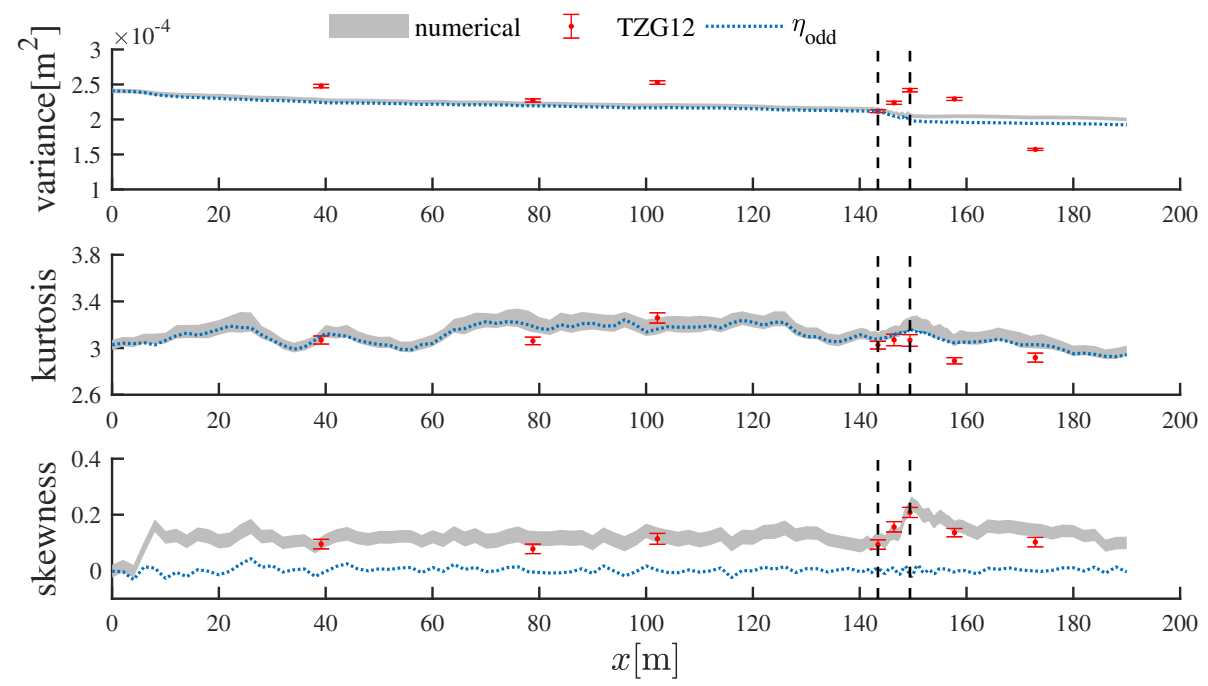

Figure 4: The variance $\left(\sigma^{2}\right)$, kurtosis $\left(\lambda_{4}\right)$ and skewness $\left(\lambda_{3}\right)$ for case A1 (case 1 in TZG12). The grey-shaded strip is the $95 \%$ confidence interval of the statistical moments obtained from our numerical simulation. The red dots and error bars are obtained from TZG12. The blue dotted line shows the linear signal $\eta_{\text {odd }}$ obtained from phase inversion. The dashed vertical lines denote the beginning and end of the slope.

\subsubsection{Maximum kurtosis and skewness and their location}

Tab. 3 gives the maximum values of the kurtosis and skewness for cases A1-A3, as well as the (coincidental) locations where both quantities reach their maxima for each case. The difference between these three cases is the peak period, which increases as we go from case A1 to A3, and thus the effective water depth $\left(k_{p 1} d_{1}\right.$ and $\left.k_{p 2} d_{2}\right)$, which becomes shallower (cf. Tab. 2). We note that, although the significant wave height is the same, the steepness is not and decreases as we go from case A1 to A3 (see Tab. 2). The larger the peak period, the larger are the maximum values of the kurtosis and skewness and the longer the lengths of latency, i.e. the distances to the right of the top of the slope where the maxima occur.

\subsubsection{Harmonic extraction}

Linearizing the signal according to the method in $§ 3.4$ gives the blue dotted lines in Figs. 4-6 (also denoted $\eta_{\text {odd }}$ ). These results indicate that the variance is mostly made up from linear terms on 

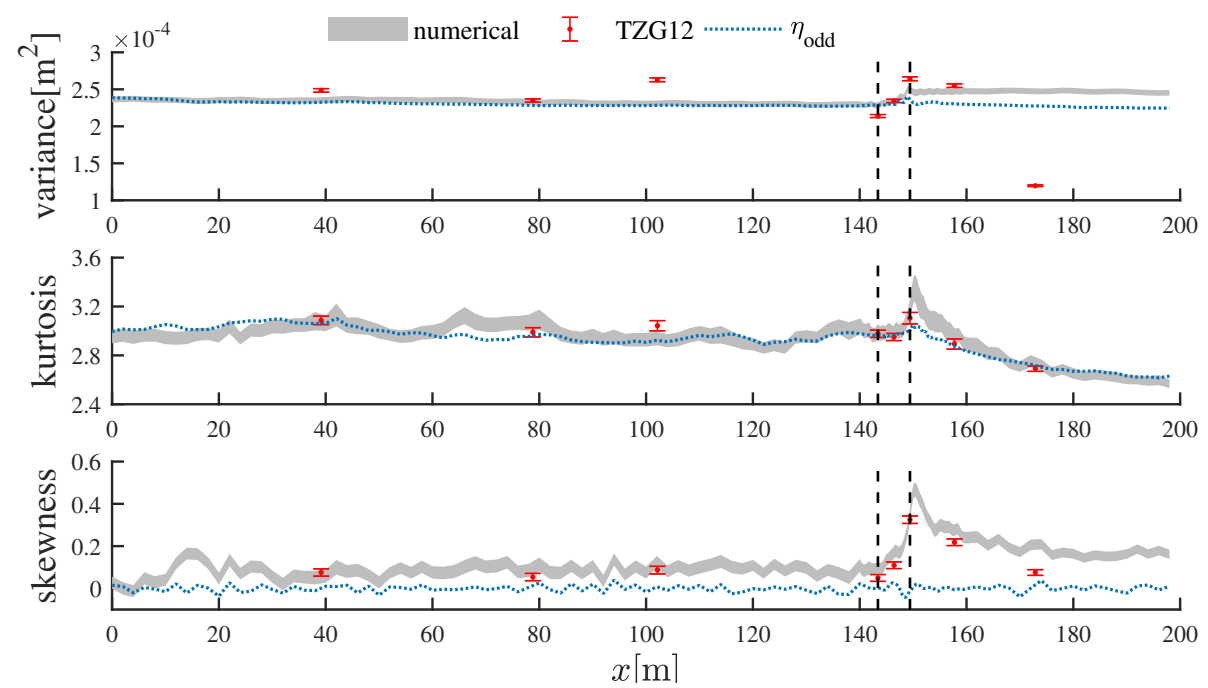

Figure 5: The variance $\left(\sigma^{2}\right)$, kurtosis $\left(\lambda_{4}\right)$ and skewness $\left(\lambda_{3}\right)$ for case A2 (case 2 in TZG12). The legend is the same as in Fig. 4.
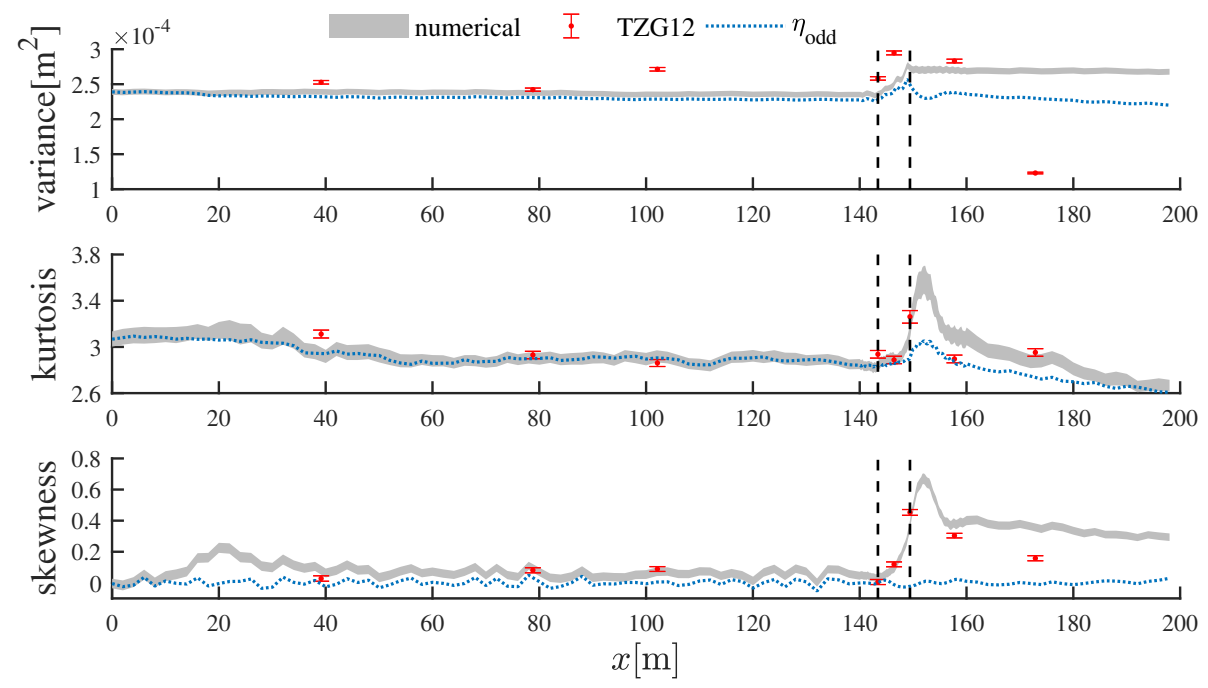

Figure 6: The variance $\left(\sigma^{2}\right)$, kurtosis $\left(\lambda_{4}\right)$ and skewness $\left(\lambda_{3}\right)$ for case A3 (case 3 in TZG12). The legend is the same as in Fig. 4.

Table 3: The position of maximum skewness and kurtosis for cases A1-A3: the effect of peak period. The end of the slope is at $x=149.41 \mathrm{~m}$.

\begin{tabular}{lccc}
\hline Case & $\mathrm{A} 1\left(T_{p}=1.27 \mathrm{~s}\right)$ & $\mathrm{A} 2\left(T_{p}=1.70 \mathrm{~s}\right)$ & $\mathrm{A} 3\left(T_{p}=2.12 \mathrm{~s}\right)$ \\
\hline Deeper depth $k_{p 1} d_{1}$ & 1.61 & 1.06 & 0.81 \\
Shallower depth $k_{p 2} d_{2}$ & 0.99 & 0.70 & 0.54 \\
Location of maximum $[\mathrm{m}]$ & 149.59 & 150.38 & 152.02 \\
Latency $\Delta x_{\mathrm{L}}[\mathrm{m}]\left(k_{p 2} \Delta x_{\mathrm{L}}\right)$ & $0.18(0.59)$ & $0.97(2.26)$ & $2.62(4.72)$ \\
Kurtosis & 3.30 & 3.33 & 3.60 \\
Skewness & 0.24 & 0.46 & 0.68 \\
\hline
\end{tabular}




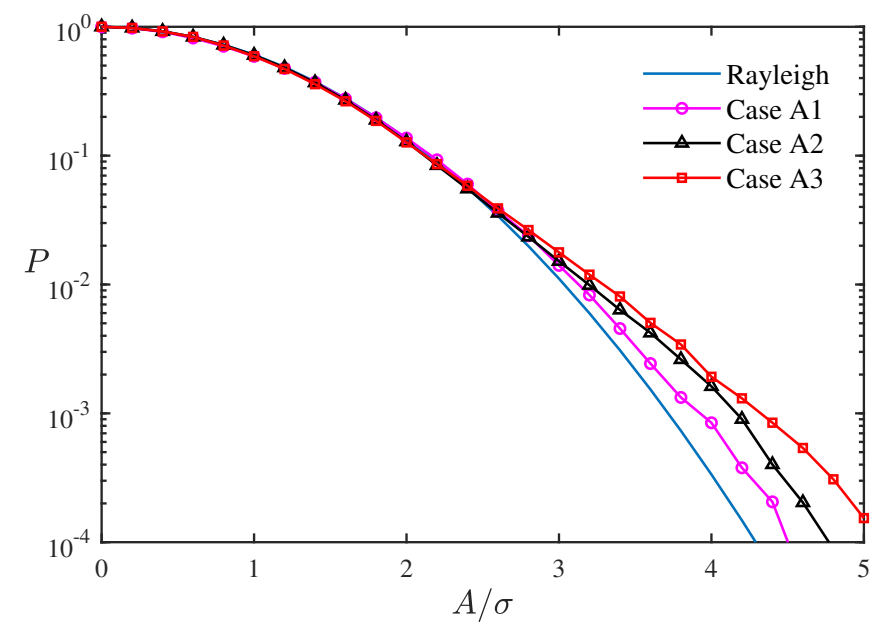

Figure 7: Exceedance probability at the locations of maximum skewness and kurtosis for different peak periods (cases A1-A3).
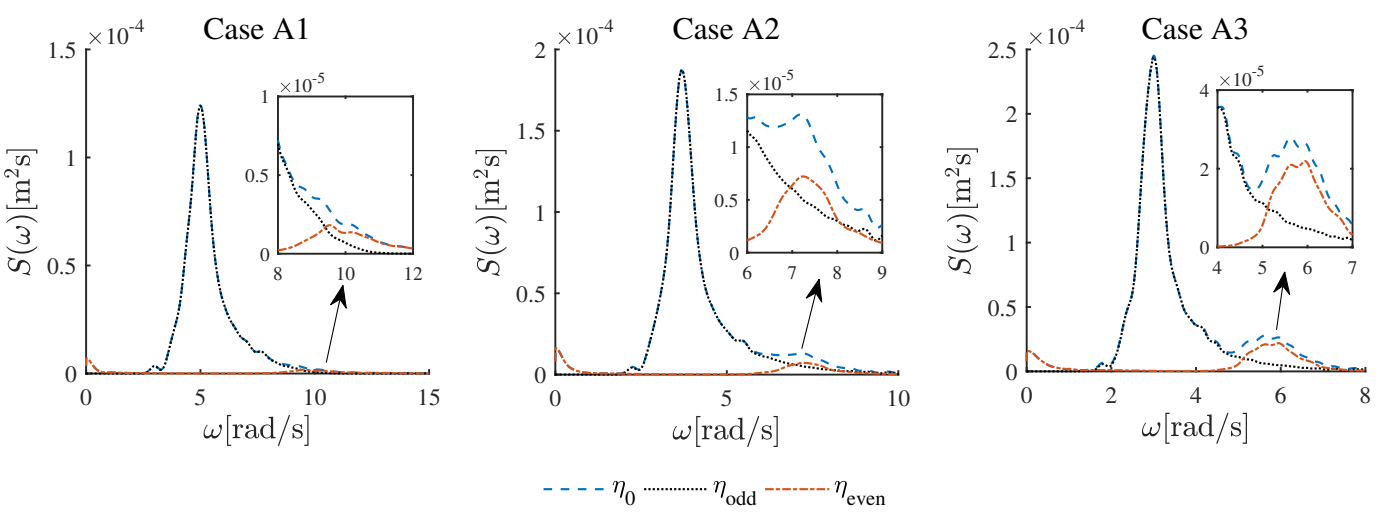

Figure 8: Energy spectra of $\eta_{0}, \eta_{\text {odd }}$ and $\eta_{\text {even }}$ for cases A1-A 3 at the locations where the skewness and kurtosis reach their maxima. The insets examine the spectra near $\omega=2 \omega_{p}$.

the deeper side, whereas the second-order terms make a greater contribution to the variance on the shallower side and increasingly so for shallower depths (i.e. smaller $k_{p 2} d_{2}$ ). On the deeper side, the kurtosis increases slightly for case $\mathrm{A} 1\left(k_{p 1} d_{1}=1.61 \geq 1.36\right.$, where 1.36 is the minimum depth, in the narrow-banded limit, for modulational instability to occur, causing the kurtosis to increase) and stays approximately constant for cases $\mathrm{A} 2\left(k_{p 1} d_{1}=1.06 \leq 1.36\right)$ and $\mathrm{A} 3\left(k_{p 1} d_{1}=0.81 \leq 1.36\right)$. On the shallower side, the kurtosis starts to decrease, which becomes more significant for smaller $k_{p 2} d_{2}$ (i.e. shallower shallow depths). These behaviours are predicted well by the linear terms on both the shallower and the deeper side, and are a sign of (slow) nonlinear evolution of the linear signal (excluding second- and higher-order (bound) waves). What is not captured by the linear terms is the rapid increase and decrease of the kurtosis near the top of the slope, which we can therefore conclude is due to second-order terms. All of the changes in the skewness, including the slight increase on the deeper side (from 0.0 at the wavemaker), the maximum near the top of the slope and the greater equilibrium value on the shallower side, are due to the second-order terms.

Fig. 8 presents the energy spectrum of $\eta_{\text {odd }}$ (the approximate linear surface elevation), $\eta_{\text {even }}$ (the approximate second-order surface elevation) and $\eta_{0}$ (the fully nonlinear surface elevation). It is evident from this figure that the energy in $\eta_{\text {even }}$ is exactly concentrated where second-order energy would be, namely at double the peak frequency (the so-called super-harmonic terms) and near zero (the sub-harmonic terms), confirming the validity of our harmonic extraction. 

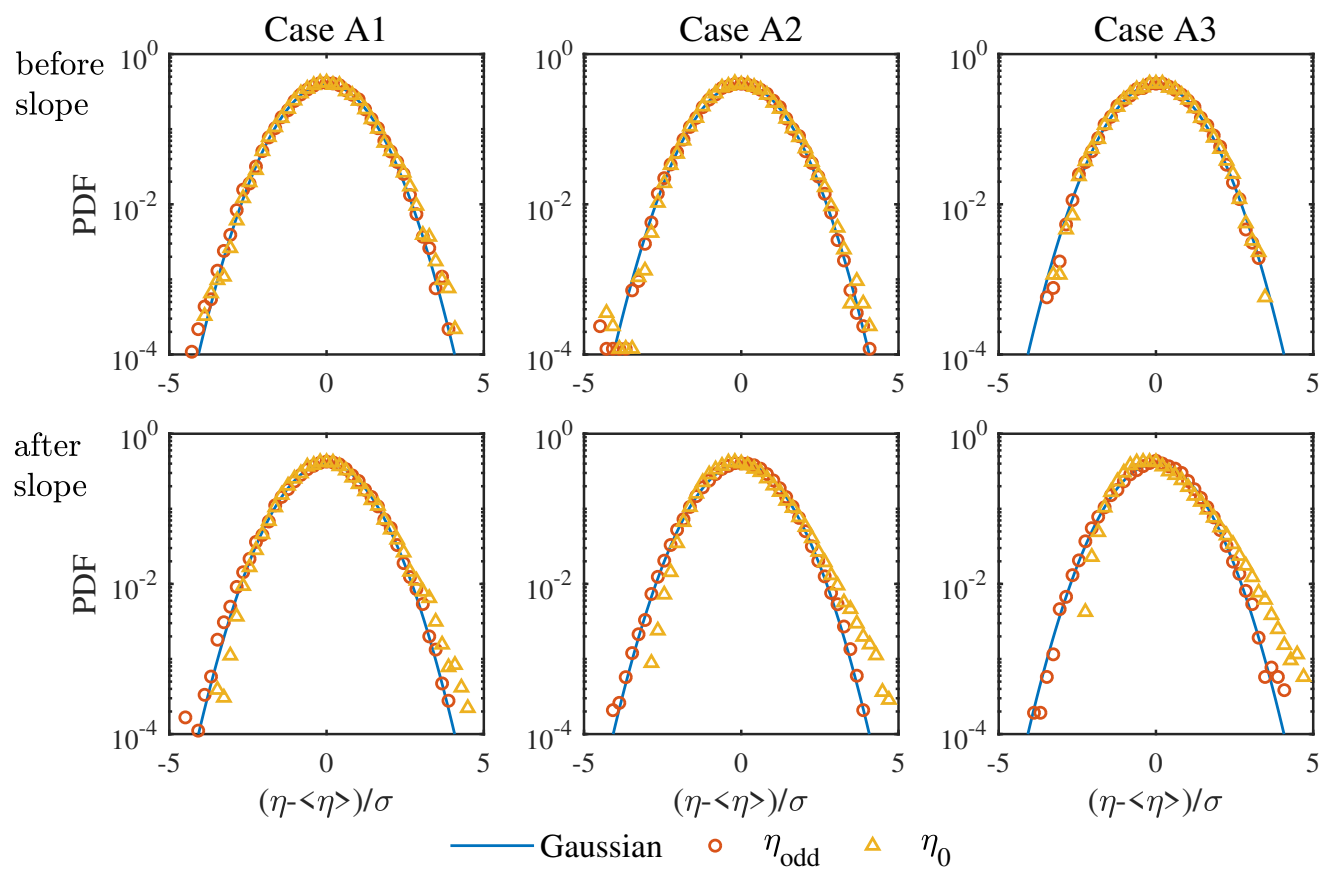

Figure 9: Probability density functions (PDF) of the normalized surface elevation for cases A1-A3 at locations just before the slope (top row) and after the slope where the skewness and kurtosis reach maxima (bottom row). The triangles denote the complete nonlinear signal $\eta_{0}$, the circles the linear signal $\eta_{\text {odd }}$ obtained from phase inversion, and the continuous line a Gaussian distribution.

\subsubsection{Probability density function}

Fig. 9 shows the probability density functions of the surface elevation for cases A1-A3 at two locations: before the slope (top row) and after the slope at the location where the skewness and kurtosis reach maxima (bottom row). Before the slope, the probability density functions of the fully nonlinear signal and the linear signal are approximately Gaussian distributed. After the slope, the linear signal remains approximately Gaussian distributed, and the skewness remains close to zero. For each case, the fully nonlinear signal has a positive skewness and deviates from a Gaussian distribution.

\subsubsection{Comparison with the literature}

Tab. 4 compares the maximum values of kurtosis and skewness we have found for cases 1-3 of TZG12 to those found therein. Before we make this comparison, we should emphasize that, due to the limited number of wave gauges, the maxima were likely not captured exactly in the experiments of TZG12. The comparison is therefore indicative, not definitive. Also shown in Tab. 4 are the values obtained using numerical solutions of the Boussinesq equations by GTZP13. As noted by these authors, the Boussinesq equations of GTZP13 overpredict (compared to the experiments of TZG12, noting the limited number of spatial observations) the maximum values for the shallower cases (A2-A3), for which the peaks are observed. Our fully nonlinear simulations still overpredict the maximum values but to a lesser extent, similar to the fully nonlinear simulations of DG17. Our fully nonlinear numerical simulations for case 3 are also in broad quantitative agreement with fully nonlinear numerical simulations by VD14 for the same case; both show a clear peak in skewness but no clear peak in kurtosis (VD14 do not consider cases 2 and 3 of TZG12). For all three cases, our fully nonlinear numerical simulations are in good quantitative agreement with fully nonlinear numerical simulations by DG17 with our values for the peaks slightly larger in magnitude. 
Table 4: Summary of maximum values of kurtosis and skewness for cases 1-3 of TZG12 in the literature.

\begin{tabular}{c|ccccc|ccccc}
\hline & \multicolumn{9}{|c}{ Kurtosis } & \multicolumn{2}{c}{ Skewness } \\
Case & TZG12 & GZTP13 & VD14 & DG17 & $\begin{array}{c}\text { This } \\
\text { paper }\end{array}$ & TZG12 & GZTP13 & VD14 & DG17 & $\begin{array}{c}\text { This } \\
\text { paper }\end{array}$ \\
\hline A1 & 3.0 & 3.0 & 3.3 & 3.3 & 3.30 & 0.2 & 0.3 & 0.2 & 0.16 & 0.24 \\
A2 & 3.1 & 3.4 & - & 3.2 & 3.33 & 0.3 & 0.5 & - & 0.35 & 0.46 \\
A3 & 3.2 & 3.9 & - & 3.4 & 3.60 & 0.5 & 0.8 & - & 0.54 & 0.68 \\
\hline
\end{tabular}

\subsection{Effect of the significant wave height}

\subsubsection{Spatial profiles of the statistical moments}

In the previous section, we have shown the peak frequency affects both the magnitude of the peak in kurtosis and skewness and what we call the length of latency, the distance to the right of the top of the slope where the maxima occur. In doing so, we have simultaneously varied the effective water depth and the steepness, despite keeping the significant wave height constant. Case A3, which has the largest peak values of kurtosis and skewness, has the largest wave length and therefore the smallest steepness $\left(k_{p i} a_{c}\right)$, whereas it also the smallest effective water depth $\left(k_{p i} d_{i}\right)$.

In Fig. 10, we examine the role of varying the significant height, thus changing the steepness, whilst leaving the effective water depth unchanged (not considered by TZG12). Shown are cases B2, B4 and B6 in Tab. 2, which have the same peak frequency but different significant wave heights. At the location of the largest kurtosis and skewness of these three cases, the exceedance probability of the Hilbert envelopes of the waves is shown in Fig. 11. Clearly, case B3 has higher exceedance probabilities for larger waves than the other two less steep cases.
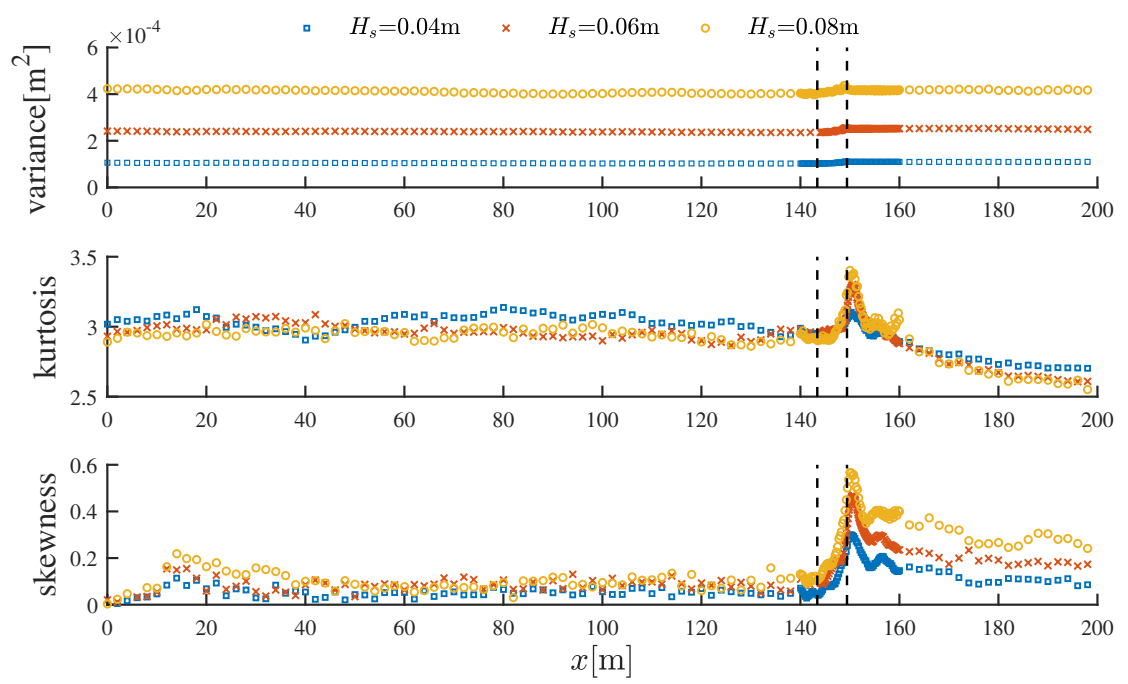

Figure 10: The variance $\left(\sigma^{2}\right)$, kurtosis $\left(\lambda_{4}\right)$ and skewness $\left(\lambda_{3}\right)$ for different significant wave heights (cases B2, B4 and B6). The dashed vertical lines denote the beginning and the end of the slope.

\subsubsection{Maximum kurtosis and skewness and their location}

In Fig. 10, it can be found that both the peaks of kurtosis and skewness become larger as the significant wave height increases. Tab. 5 gives the maximum values of the kurtosis and skewness for cases B2, B4 and B6, as well as the (coincidental) locations where both quantities reach their maxima for each case. Compared to the effect of peak frequency (cf. Tab. 3), the significant wave height has a small effect on the location of the maxima, but does affect their magnitudes. This is consistent with second-order terms being the cause of these maxima. 


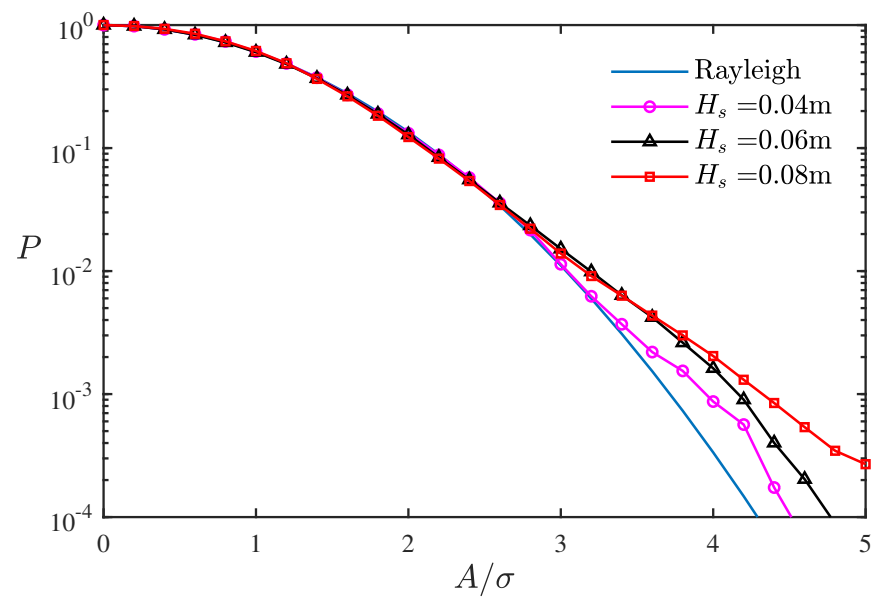

Figure 11: Exceedance probability of the amplitude of the Hilbert envelope of the free surface at the locations of maximum skewness and kurtosis for cases B2 $\left(H_{s}=0.04 \mathrm{~m}\right), \mathrm{B} 4\left(H_{s}=0.06 \mathrm{~m}\right)$ and $\mathrm{B} 6\left(H_{s}=0.08 \mathrm{~m}\right)$.

Table 5: The position of maximum skewness and kurtosis for cases B1-B6: the effect of significant wave height. The end of the slope is at $x=149.41 \mathrm{~m}$.

\begin{tabular}{lcccccc}
\hline Case & B1 & B2 & B3 & B4 & B5 & B6 \\
$H_{s}[\mathrm{~m}]$ & 0.03 & 0.04 & 0.05 & 0.06 & 0.07 & 0.08 \\
\hline Steepness $k_{p 1} a_{c 1}$ & 0.019 & 0.025 & 0.031 & 0.038 & 0.044 & 0.050 \\
Latency $\Delta x_{\mathrm{L}}[\mathrm{m}]$ & 1.08 & 1.04 & 0.99 & 0.97 & 0.98 & 0.95 \\
$\left(k_{p 2} \Delta x_{\mathrm{L}}\right)$ & $(2.52)$ & $(2.43)$ & $(2.31)$ & $(2.26)$ & $(2.29)$ & $(2.22)$ \\
Kurtosis & 2.95 & 3.09 & 3.22 & 3.33 & 3.27 & 3.40 \\
Skewness & 0.26 & 0.30 & 0.37 & 0.46 & 0.53 & 0.58 \\
\hline
\end{tabular}




\subsubsection{Scaling analysis and comparison with the literature}

To examine this further, Fig. 12 performs a scaling analysis of the maximum values of skewness $\lambda_{3}$ and excess kurtosis $\lambda_{4}-3$ with respect to steepness. It is evident from the lines fitted to the data that skewness $\lambda_{3}$ and kurtosis $\lambda_{4}$ appear to scale linearly with steepness, although the evidence for skewness is more conclusive. Although no explicit scaling analysis was performed therein, our findings in this section are not inconsistent with GTZP13, who examined the effect of wave steepness using the Boussinesq equations. GTZP13 also conclude the peaks in skewness and kurtosis are associated with second-order terms.
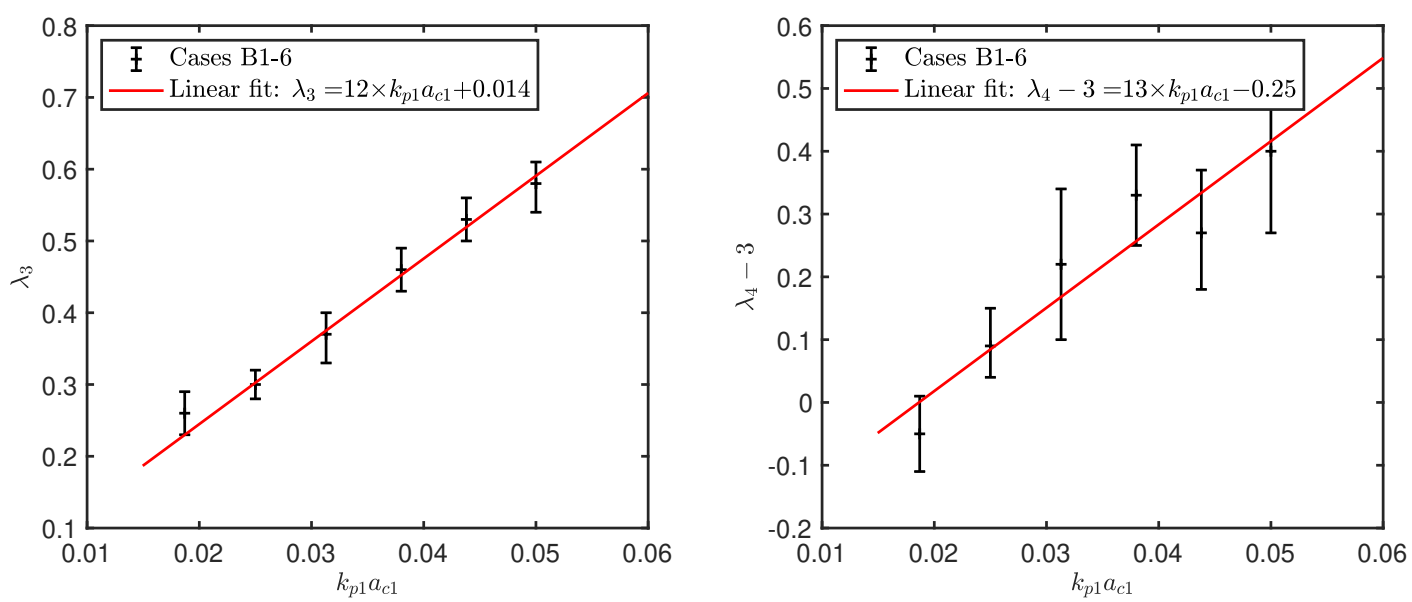

Figure 12: Scaling analysis of the maximum values of skewness $\lambda_{3}$ and excess kurtosis $\lambda_{4}-3$ with respect to steepness $k_{p 1} a_{c 1}$.

\subsection{Effect of the length of the slope}

So far, we examined the effect of peak period and significant wave height for the same sloping bathymetry as in TZG12 (a slope of 1:20). In this subsection, we will vary the slope by changing its (horizontal) length, keeping the depth of the deep side $(0.6 \mathrm{~m})$ and the shallow side $(0.3 \mathrm{~m})$ and the input irregular wave signal unchanged. As in the previous cases, the kurtosis and skewness abruptly increase near the end of sloping bottom, reaching maximum values at a location a short distance after the slope before abruptly decreasing again. We vary the horizontal length of slope between 0 and $24 \mathrm{~m}$, as shown in Tab. 2 (cases C1-C7).

\subsubsection{Maximum kurtosis and skewness and their location}

For different slopes, Tab. 6 shows values of the maximum kurtosis and skewness, as well at the locations where these maxima occur. As the length of the slope decreases, the peak values of kurtosis and skewness increase with the largest values occurring for a step. The length of latency, the distance to the right of the top of the slope where the maxima occur, increases as the length of the slope decreases. For the mildest slope we have considered, i.e. case $\mathrm{C} 7$ with a length of slope of $L_{s}=24 \mathrm{~m}$, the location of the peak in kurtosis and skewness is very close to the end of the slope. We emphasize that as the length of the slope increases, the peaks gradually disappear, making them almost unrecognizable as peaks for the mildest slope we consider. That is, for case C7 with a length of slope of $L_{s}=24 \mathrm{~m}$, the peak values of kurtosis (3.06) is almost the same as that of the input signal (3.0). For the infinitely steep slope or step, i.e. case C1 with a length of slope of $L_{s}=0 \mathrm{~m}$, there is a relatively long latency of $2.20 \mathrm{~m}$. The kurtosis peaks at $\lambda_{4}=3.61$ : an excess kurtosis that is 10 times larger than for the mildest slope.

\subsubsection{Exceedance probability}

The distributions of the exceedance probability at the locations of maximum skewness and kurtosis are shown in Fig. 13. As the length of the slope increases, the exceedance probability of a given surface elevation Hilbert envelope amplitude $A / \sigma$ becomes lower. When the slope is relatively 
Table 6: The position of maximum skewness and kurtosis for cases C1-C7: the effect of slope.

\begin{tabular}{cccccccc}
\hline Case $\left(L_{s}[\mathrm{~m}]\right)$ & $\mathrm{C} 1(0.0)$ & $\mathrm{C} 2(1.5)$ & $\mathrm{C} 3(3.0)$ & $\mathrm{C} 4(6.0)$ & $\mathrm{C} 5(12.0)$ & C6 (18.0) & C7 (24.0) \\
\hline Deeper depth $k_{p 1} d_{1}$ & 1.06 & 1.06 & 1.06 & 1.06 & 1.06 & 1.06 & 1.06 \\
Shallower depth $k_{p 2} d_{2}$ & 0.70 & 0.70 & 0.70 & 0.70 & 0.70 & 0.70 & 0.70 \\
End of slope[m] & 143.41 & 144.91 & 146.41 & 149.41 & 155.41 & 161.41 & 167.41 \\
Location of maximum $[\mathrm{m}]$ & 145.61 & 146.57 & 147.85 & 150.38 & 156.31 & 162.15 & 167.77 \\
Latency $\Delta x_{\mathrm{L}}[\mathrm{m}]$ & 2.20 & 1.66 & 1.44 & 0.97 & 0.90 & 0.74 & 0.33 \\
$\left(k_{p 2} \Delta x_{\mathrm{L}}\right)$ & $(5.13)$ & $(3.87)$ & $(3.36)$ & $(2.26)$ & $(2.10)$ & $(1.73)$ & $(0.77)$ \\
Kurtosis & 3.61 & 3.43 & 3.42 & 3.33 & 3.20 & 3.11 & 3.06 \\
Skewness & 0.61 & 0.56 & 0.53 & 0.46 & 0.40 & 0.36 & 0.31 \\
\hline
\end{tabular}

steep $\left(L_{s} \leq 6 \mathrm{~m}\right)$, the distributions become indistinguishable except for a deviation in the tail. When the slope is sufficiently mild $\left(L_{s}=24 \mathrm{~m}\right)$, the distribution is close to a Rayleigh distribution except for a deviation in the tail. For four specific values of $A / \sigma$, the exceedance probabilities as a function of the horizontal slope length $L_{s}$ are shown in Fig. 14. As the length of the slope increases, the exceedance probability decreases and tends to that predicted by a Rayleigh distribution. The probabilities increase rapidly for steep slopes (smaller $\left.L_{s}\right)$, with the step $\left(L_{s}=0\right)$ providing an upper limit.
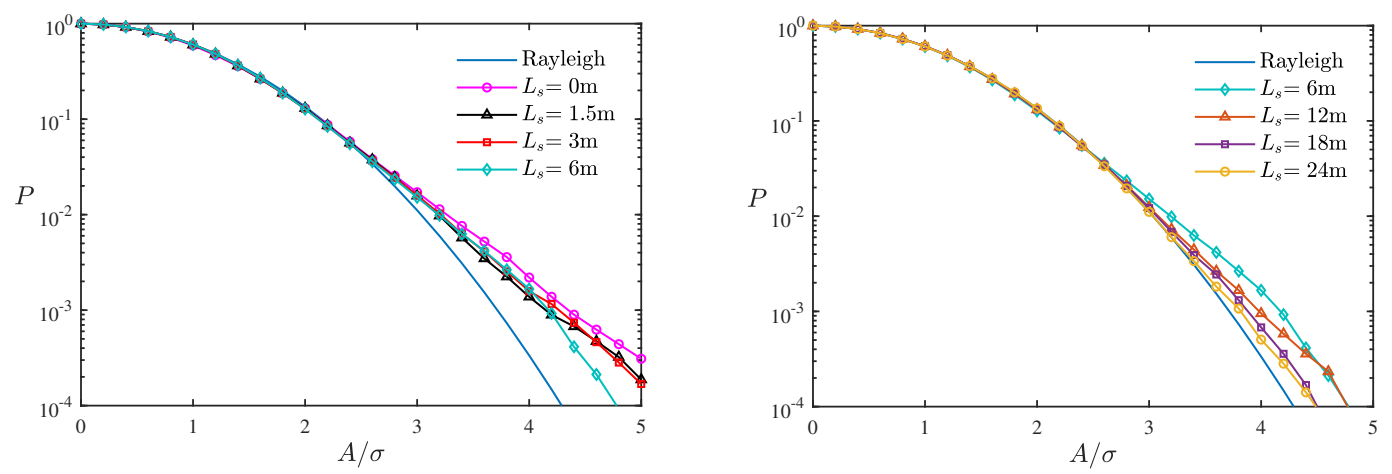

Figure 13: Exceedance probability of the amplitude of the Hilbert envelope of the free surface at the locations of maximum skewness and kurtosis for different slopes (cases C1-C7). The solid line without markers denotes the Rayleigh distribution.

\subsubsection{Comparison with the literature}

Through numerical simulations of the Boussinesq equations, GTZP13 have also found that the largest values of the peaks in kurtosis and skewness occur when the slope was largest. They only considered three different slopes, and limitations on their Boussinesq equations meant they could not consider a step. GTZP13 also found that for sufficiently shallow water and sufficiently large slope length the local maxima of the statistical moments disappeared.

\subsection{Effect of the depth of shallower side}

\subsubsection{Spatial profiles of the statistical moments}

We now vary the depth of the shallower side $d_{2}$ and thus the slope, while keeping other parameters unchanged. In cases D1 to D3, $d_{2}$ is respectively $0.3 \mathrm{~m}, 0.35 \mathrm{~m}$ and $0.4 \mathrm{~m}$ (see Tab. 2). Fig. 15 shows the variance, kurtosis and skewness for these three cases. Before the slope, the value of the variance of case D1 $\left(d_{2}=0.3 \mathrm{~m}\right)$ is larger than the other two cases (cases D2 and D3), probably as a result of reflections from the slope. Apart from this, the behaviour is qualitatively similar to the previous cases. As the depth of the shallower side $d_{2}$ decreases, the peak in the kurtosis and skewness near the top of the slope increases. Fig. 15 shows the distributions of the exceedance probability at the locations of maximum skewness and kurtosis. 

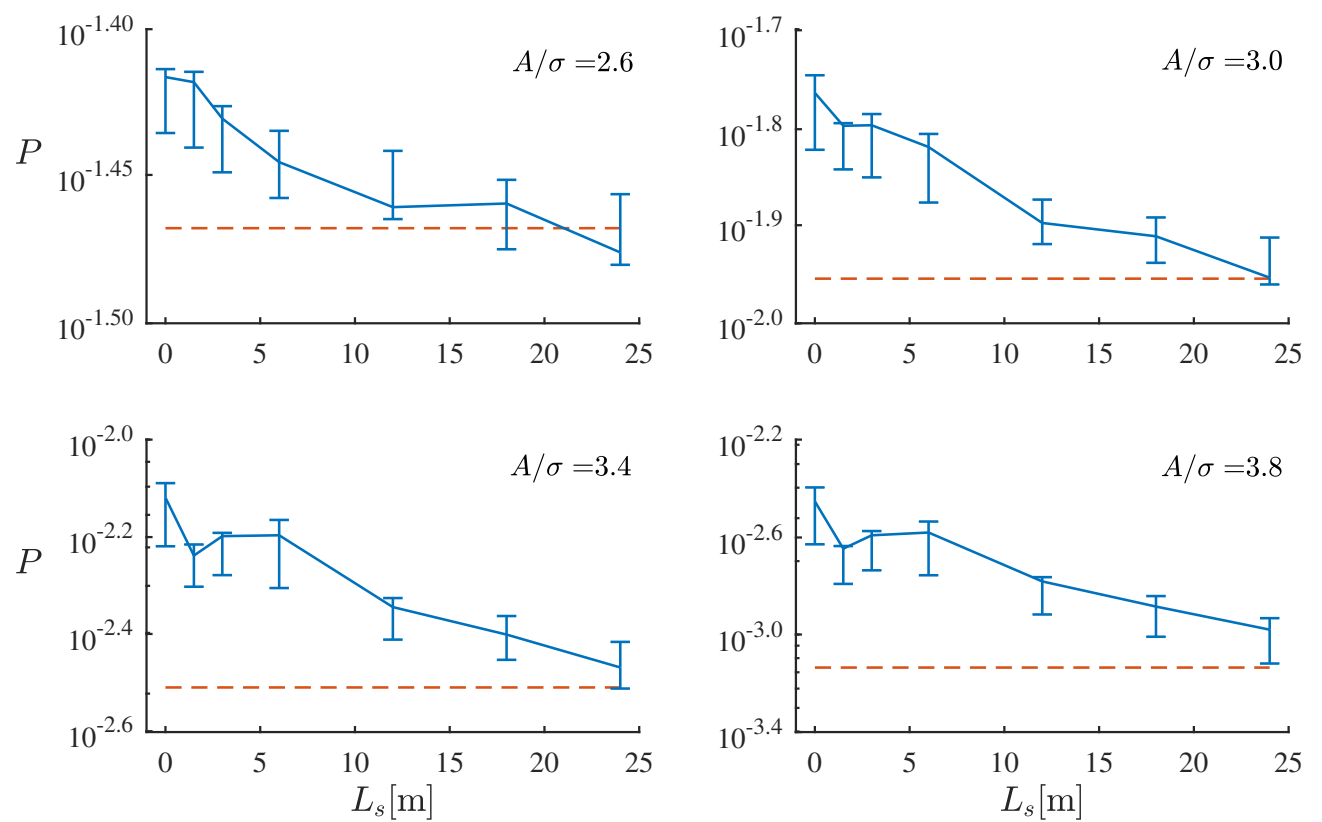

Figure 14: Exceedance probability of the amplitude of the Hilbert envelope of the free surface for four values of $A / \sigma$, where $A$ is the wave envelop. The error bar presents a $95 \%$ confidence interval of the exceedance probability obtained from bootstrapping. The horizontal red dashed line is the exceedance probability of a Rayleigh distribution for the given value of $A / \sigma$.
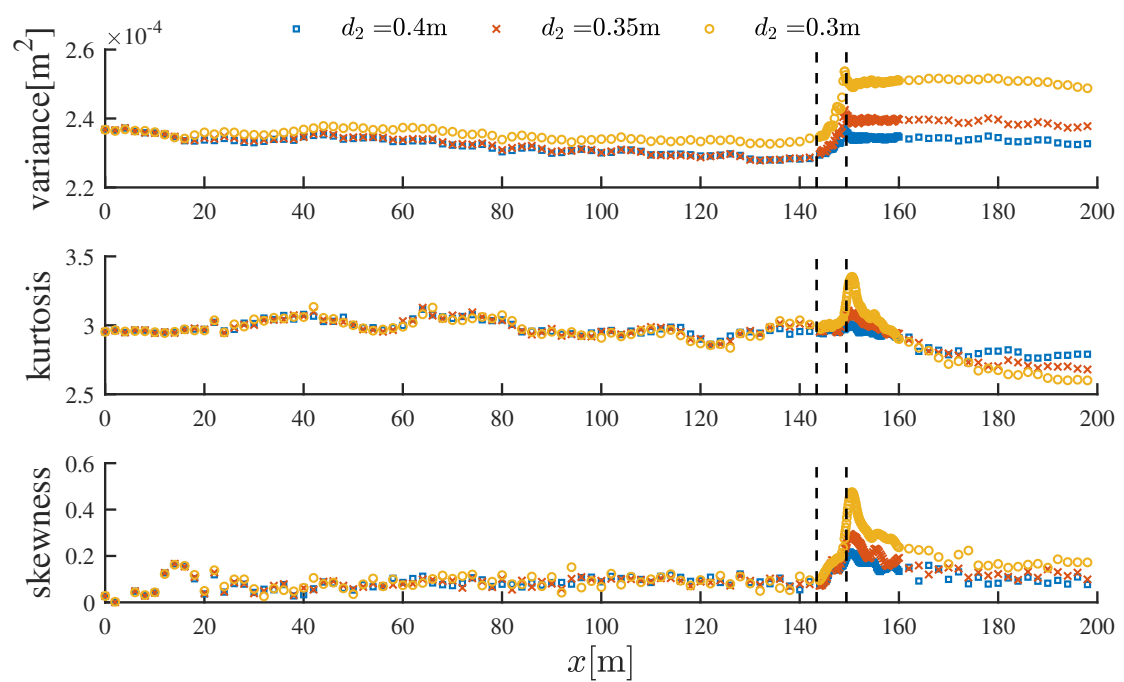

Figure 15: The variance $\left(\sigma^{2}\right)$, kurtosis $\left(\lambda_{4}\right)$ and skewness $\left(\lambda_{3}\right)$ for different shallower side depths (cases D1-D3). The dashed vertical lines denote the beginning and the end of the slope. 


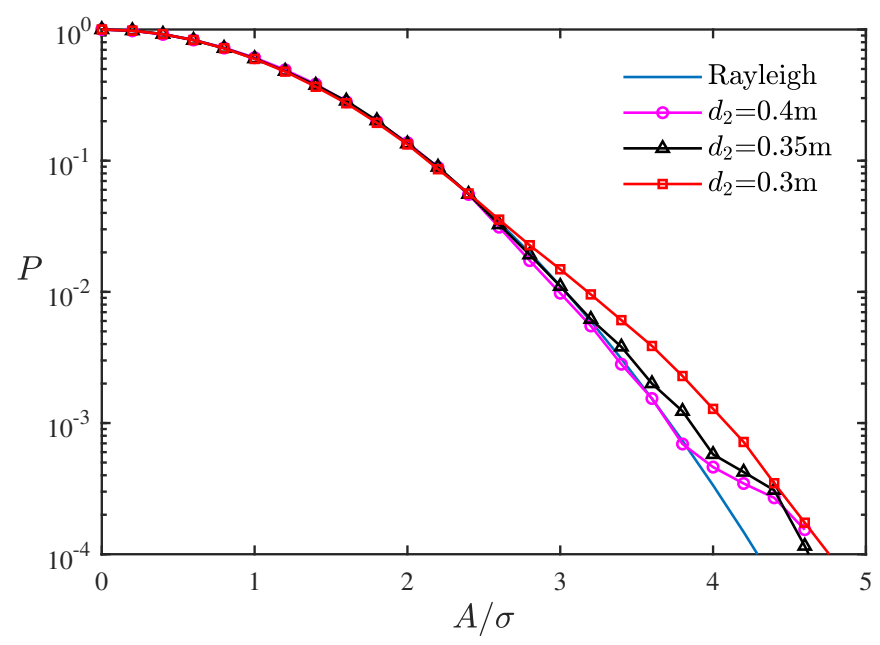

Figure 16: Exceedance probability of the amplitude of the Hilbert envelope of the free surface at the locations of maximum skewness and kurtosis for cases D1-D3. The solid lines without markers denote the Rayleigh distribution.

\subsubsection{Maximum kurtosis and skewness and their location}

Tab. 7 gives the maximum values of the kurtosis and skewness for cases D1-D3, as well as the (coincidental) locations where both quantities reach their maxima for each case. The length of latency is relatively insensitive to varying the depth of the shallower side. We note that we do not employ a breaking model in our numerical wave tank. When wave breaking happens, the simulation will simply stop and we thus have to avoid it. For simulations with $d_{2}$ smaller than 0.25 $\mathrm{m}$, wave breaking always occurs, and we therefore have not investigated such shallow depths. To prevent breaking at shallow depths, we could evidently have reduced the amplitude of the waves. To avoid varying more than one variable at the same time, we have not done so here.

Table 7: The position of maximum skewness and kurtosis for cases D1-D3: the effect of the depth of the shallow side.

\begin{tabular}{lccc}
\hline Case & $\mathrm{D} 1\left(d_{2}=0.3 \mathrm{~m}\right)$ & $\mathrm{D} 2\left(d_{2}=0.35 \mathrm{~m}\right)$ & $\mathrm{D} 3\left(d_{2}=0.4 \mathrm{~m}\right)$ \\
\hline Deeper depth $k_{p 1} d_{1}$ & 1.06 & 1.06 & 1.06 \\
Shallower depth $k_{p 2} d_{2}$ & 0.70 & 0.76 & 0.82 \\
Location of maximum $[\mathrm{m}]$ & 150.38 & 150.43 & 150.58 \\
Latency $\Delta x_{\mathrm{L}}[\mathrm{m}]\left(k_{p 2} \Delta x_{\mathrm{L}}\right)$ & $0.97(2.26)$ & $1.02(2.58)$ & $1.17(3.20)$ \\
Kurtosis & 3.33 & 3.08 & 3.00 \\
Skewness & 0.46 & 0.29 & 0.21 \\
\hline
\end{tabular}

\subsubsection{Comparison with the literature}

Taking case 1 of TZG12 as their (approximate) base case $\left(k_{p 1} d_{1}=1.8\right.$ on the deeper side vs. $k_{p 1} d_{1}=1.06$ in our case), VD14 have also examined the effect of lowering the depth of the shallower side (to $k_{p 2} d_{2}=0.78-1.03$ vs. $k_{p 1} d_{1}=0.70-0.82$ in our case) and have found qualitatively similar results, namely an increase in the magnitudes of the peaks in skewness and kurtosis. Using fully nonlinear simulations, VD14 found very considerable peaks in skewness (up to $\lambda_{3}=0.9$ ) and kurtosis (up to $\lambda_{4}=4.4$ ). These are larger than ours, but the different relative depths and steepnesses make direct comparison impossible. Qualitatively similar results have been obtained using numerical simulations of the Boussinesq equations by GTZP13 and experimentally by TRJR20. 


\section{Conclusion}

In this paper, we have studied the occurrence of extreme waves when random surface gravity waves propagate over a strong depth transition. We have done so through numerical simulations of unidirectional, nonlinear, irregular waves using the Fast Multipole Boundary Element Method (FMBEM), which proved capable of solving this computationally intensive problem efficiently. To achieve this, we have extended the numerical wave tank developed by [19] using the Fast Multipole Method to make it efficient enough to deal with random simulations. A convergence study has been carried out to ensure the accuracy of our simulations. The results of our simulations conform well with the experimental results of Trulsen et al. [32] (TZG12), validating the method we have employed. Compared to these experiments, we have been able to study in more detail the maximum values of skewness and kurtosis at the top of steep slopes, as well as the exact location where these maxima occur. Like Gramstad et al. [13] (GTZP13), who did so using numerical simulations of the Boussinesq equations, we have examined the effect of varying the peak period, significant wave height, the length of the slope and the depth of the shallower side. Compared to GTZP13, we have considered a greater range of slopes, including a step or infinitely steep slope.

Through harmonic separation [2], we have shown that, to leading-order, the second-order terms are responsible for the abrupt peaks in skewness and kurtosis at the top of the slope, noting that both peaks always coincide. A similar conclusion has previously been reached by GTZP13. We hypothesize that these second-order terms are made up from bound waves and free waves that are released by the interaction of the bound waves with the slope (cf. [3]). Changing the peak period, and thus the effective water depth, changes both the magnitudes of the peaks in skewness and kurtosis and the location at which they occur. For an increased peak period $T_{p}$, and thus a reduced effective water depth, the peak kurtosis and skewness are larger. The length of latency, i.e. the distance to the right of the top of the slope where the maxima occur, also increases. Increasing the significant wave height $H_{s}$ increases the peak values, but leaves the location of the peaks more or less unchanged, providing additional support for our finding that second-order terms are responsible for the peaks. As we increase the length of the slope, making it more mild, the peaks in kurtosis and skewness start to disappear, the length of latency reduces, and the exceedance probability becomes comparable to the Rayleigh distribution. The largest peaks and the longest lengths of latency occur when the slope becomes a step. Finally, the peaks in kurtosis and skewness become more pronounced when the depth of the shallower side is smaller, and the depth transition is greater.

We emphasize that Viotti \& Dias [33] (VD14) and Ducrozet \& Gouin [7] (DG17) have previously examined the same problem using fully nonlinear numerical simulations. VD14 focused on case 1 of TZG12 instead of case 2 as a benchmark and examined the effect of varying the depth of the shallower side, as in our cases D1-D3. The results of both VD14 and DG17 agree with ours, although we have examined a broader range of depth configurations, ranging from a mild to an infinitely steep slope (a step). VD14 have ascribed the occurrence of sharp peaks in kurtosis and skewness to the transition that occurs between the two statistical equilibria on either side of the slope. Two mechanisms occur when waves propagate over a depth transition. On the one hand, the weakly nonlinear signal needs to adjust. This would be captured by a third-order evolution equation and reflects the transition from modulationally unstable behaviour in deeper water $(k h>1.36)$ to modulationally stable behaviour in shallower water $(k h>1.2)$, as studied in Zeng \& Trulsen [36] using the nonlinear Schrödinger equation for relatively deep water. On the other hand, the bound waves at second-order need to adjust, giving rise to the peaks in skewness and kurtosis for relatively shallow depths, such as the experiments of TZG12. We show here that what VD14 call 'out-of-equilibrium dynamics' is the second mechanism. The evidence for this is threefold: (1) the peaks disappear upon linearization using harmonic separation, (2) the terms responsible for the peaks have sub- and super-harmonic frequencies second-order terms normally have (cf. Fig. 8), and (3) the length of latency is insensitive to steepness (cf. Tab. 5), suggesting a linearly propagating mechanism is responsible. This finding is consistent with Zhang et al. [37] (ZBKCH19), who form their hypothesis through bi-spectral analysis (since ZBKCH19 perform bi-spectral analysis in frequency space only, they cannot distinguish free from bound waves at second order). The sharp peaks can thus arise even when the two equilibria of the underlying linear signal, which acts as the random seed, are relatively similar despite the change in depth (cf. Figs. 4-6), provided the bound second-order harmonics are substantially different on both sides.

We foresee future work in two directions. First, we would like to derive expressions for the 
second-order (in amplitude or steepness) bound waves and their release into free waves in the limiting case of wave propagation over a step to confirm our hypothesis. Second, we would like to introduce a wave breaking model in our numerical wave tank, which presently cannot handle transition into very shallow depths, where waves start to break, but the peaks in kurtosis and skewness may nevertheless be highest.

\section{Acknowledgements}

This work has been supported by the National Natural Science Foundation of China (51979162) and the NSFC-EPSRC-NERC Grant (51479114, EP/R007632/1). TSvdB acknowledges a Royal Academy of Engineering Research Fellowship.

\section{A Appendix: Convergence study}

\section{A.1 Mesh convergence}

To demonstrate the convergence of our numerical simulations, we examine case A2 (see Tab. 2) using four levels of mesh discretization. To define the mesh density, we use the number of boundary elements per $\lambda_{\min }$, where $\lambda_{\min }$ is the smallest wave length and corresponds to the upper-bound cutoff of the frequency spectrum (i.e., $3 \omega_{p}$ ). In this convergence study, we examine mesh densities of 10, 15, 20 and 25 and label these simulations mesh 10, mesh 15, mesh 20, and mesh 25, respectively.

\section{A.1.1 Records of large waves}

We begin by examining records of large waves on a case-by-case basis. Fig. 17 shows partial time series at the locations of three wave probes used in TZG12. We choose the locations of probes 4, 5 and 6 , which are located at the start, middle and end of the slope bottom, respectively. Fig. 18 shows the partial spatial profiles of the free surface for different mesh densities at the location of the slope. In both figures, we show a series of large waves and we can conclude from both figures that convergence is obtained for the higher mesh densities: the results of mesh 20 and mesh 25 are almost the same.

\section{A.1.2 Maximum kurtosis and skewness and their location}

Although the location of the crest in Fig. 18 remains slightly sensitive to mesh density even at the finest mesh size we adopt (mesh 25), the statistical properties have clearly converged. To demonstrate this, Tab. 8 and Tab. 9 give the kurtosis and skewness at the locations of the eight probes in TZG12, which show no changes between mesh 20 and mesh 25. Tab. 10 confirms convergence has also been obtained for the maximum values of kurtosis and skewness, as well as their locations.

Table 8: Kurtosis at the locations of the eight probes in TZG12 for different mesh densities.

\begin{tabular}{ccccccccc}
\hline Probe & 1 & 2 & 3 & 4 & 5 & 6 & 7 & 8 \\
\hline mesh 10 & 3.07 & 3.06 & 2.95 & 2.98 & 2.98 & 3.19 & 3.00 & 2.73 \\
mesh 15 & 3.06 & 3.09 & 2.93 & 2.98 & 2.99 & 3.19 & 2.96 & 2.74 \\
mesh 20 & 3.05 & 3.08 & 2.94 & 2.99 & 2.99 & 3.19 & 2.97 & 2.74 \\
mesh 25 & 3.05 & 3.08 & 2.94 & 2.99 & 2.99 & 3.19 & 2.97 & 2.74 \\
\hline
\end{tabular}

Table 9: Skewness at the locations of the eight probes in TZG12 for different mesh densities.

\begin{tabular}{ccccccccc}
\hline Probe & 1 & 2 & 3 & 4 & 5 & 6 & 7 & 8 \\
\hline mesh 10 & 0.09 & 0.07 & 0.10 & 0.06 & 0.14 & 0.32 & 0.25 & 0.20 \\
mesh 15 & 0.08 & 0.07 & 0.12 & 0.09 & 0.16 & 0.36 & 0.27 & 0.21 \\
mesh 20 & 0.08 & 0.07 & 0.13 & 0.09 & 0.17 & 0.35 & 0.28 & 0.22 \\
mesh 25 & 0.08 & 0.07 & 0.13 & 0.09 & 0.17 & 0.35 & 0.28 & 0.22 \\
\hline
\end{tabular}



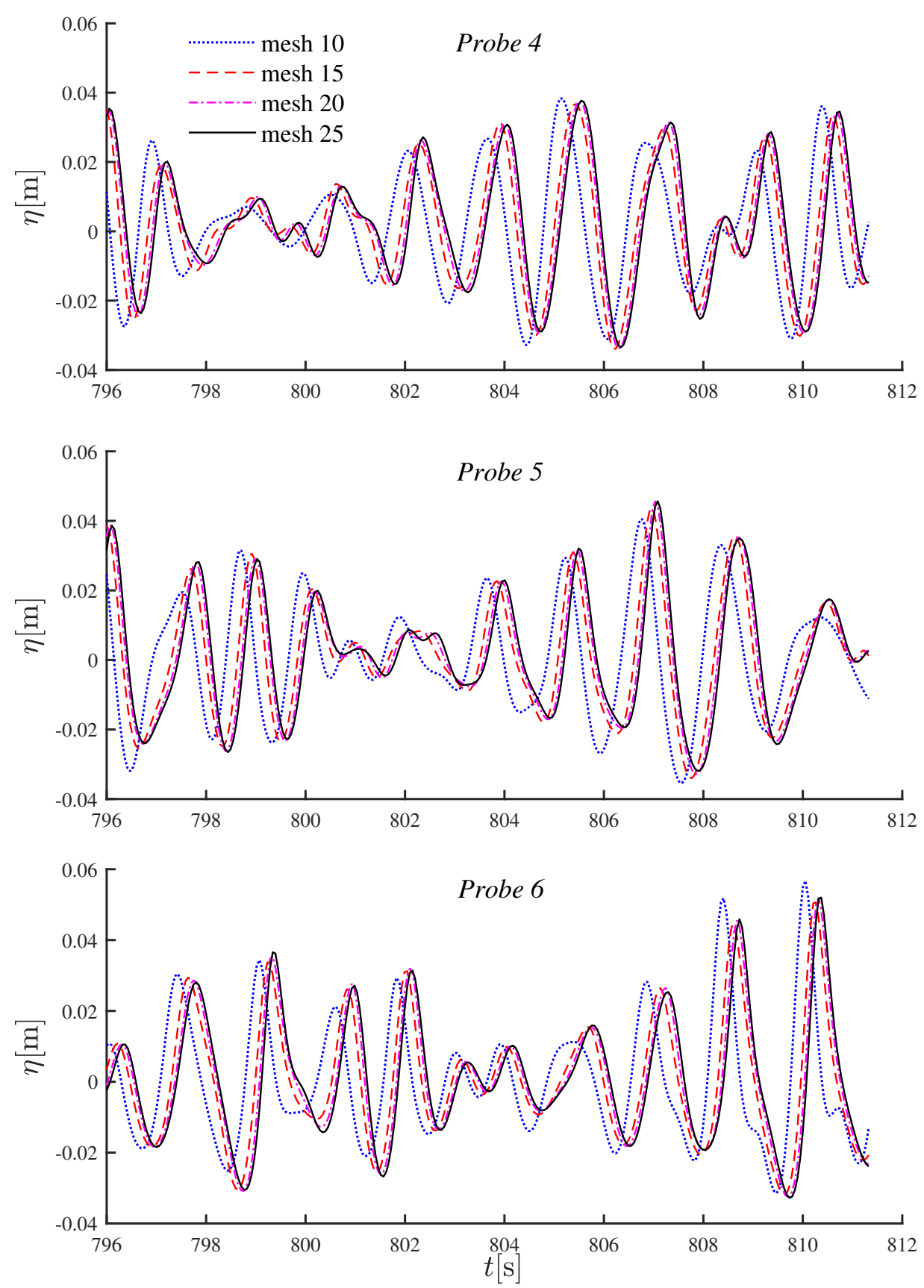

Figure 17: Partial time series of the surface elevation for different mesh densities at probes 4,5 and 6 , which are located at $143.41 \mathrm{~m}, 146.43 \mathrm{~m}$ and $149.41 \mathrm{~m}$, respectively. The time window is chosen to be $\mathrm{t}=795.0-811.3 \mathrm{~s}$, when large waves propagate over the probes.

Table 10: Convergence study of the maximum skewness and kurtosis and their location.

\begin{tabular}{lccccc}
\hline Mesh & 10 & 15 & 20 & 25 & 25 \\
$M$ & 200 & 200 & 200 & 200 & 300 \\
\hline Location of maximum $[\mathrm{m}]$ & 149.77 & 150.36 & 150.39 & 150.38 & 150.42 \\
Latency $\Delta x_{\mathrm{L}}[\mathrm{m}]$ & 0.36 & 0.95 & 0.98 & 0.97 & 1.01 \\
Kurtosis & 3.34 & 3.31 & 3.33 & 3.33 & 3.31 \\
Skewness & 0.49 & 0.46 & 0.46 & 0.46 & 0.44 \\
\hline
\end{tabular}




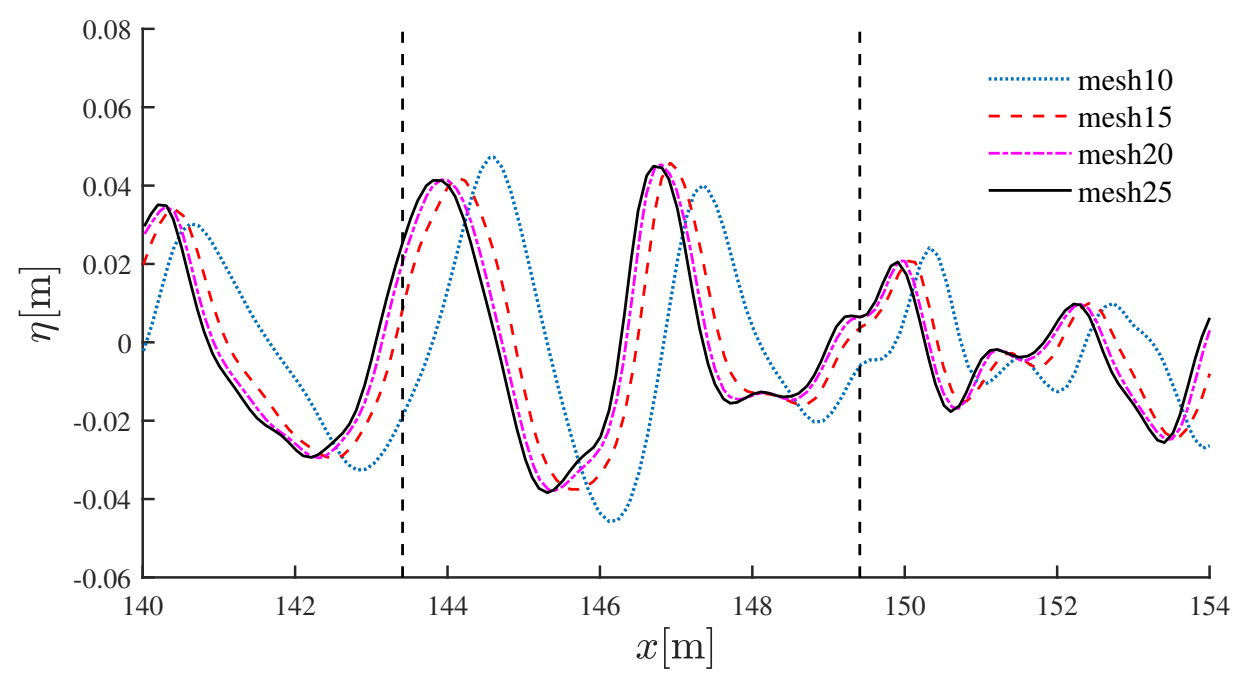

Figure 18: Partial spatial profiles of the surface elevation for different mesh densities at $t=876.1 \mathrm{~s}$, chosen so that large waves propagate through this zone at this time. The interval of $x$ is chosen to be $140-155 \mathrm{~m}$, covering the sloped bottom and two regions of constant depth it connects. The dashed vertical lines denote the beginning and the end of the slope.

\section{A.2 Random wave generation}

\section{A.2.1 Statistical moments}

We first examine the effect of the length of time over which we estimate statistical moments on the values of these moments, as shown in Fig. 19 for case A2, mesh 25 and at probe 6 (just to the left of the slope, at $x=149.41 \mathrm{~m})$. The variance, kurtosis and skewness reach steady values after approximately $t / T_{p}=900(t=1500 \mathrm{~s})$, which we then use to compute the values reported in the paper. We have performed a similar convergence study for the other cases in the paper. We emphasize that increasing the duration of the signal whilst keeping the number of random components fixed cannot ensure statistical convergence. To address this, we next increase the number of random wave components.

\section{A.2.2 Number of random wave components}

The irregular wave input signal is presented as a superposition of $M$ linear wave components. To check of $M=200$ is adequate, a simulation in which the number of components $M=300$ is carried out. Except for $M$, other parameters are set the same as in case A2 (case 2 in TZG12) and we use mesh 25. Then, the statistical moments are calculated with $95 \%$ confidence intervals obtained from bootstrapping. The result is shown in Fig. 20. For variance, kurtosis and skewness, the moments for $M=200$ and $M=300$ are quite close, especially in the area near the sloping bottom, where our interest lies. The final column in Tab. 10 confirms the effect of increasing the number of random components to $M=300$ on the maximum values of kurtosis and skewness and their locations is small. Therefore, $M=200$ is sufficient for our purposes.

\section{References}

[1] T. A. A. Adcock and P. H. Taylor. The physics of anomalous ('rogue') ocean waves. Rep. Prog. Phys., 465:3361-3381, 2014.

[2] T. E. Baldock, C. Swan, and P. H. Taylor. A laboratory study of nonlinear surface waves on water. Philos. T. Roy. Soc. A, 354:649-676, 1996.

[3] S. Beji and J.A Battjes. Experimental investigation of wave propagation over a bar. Coastal Engineering, 19(1-2):151-162, 1993. 

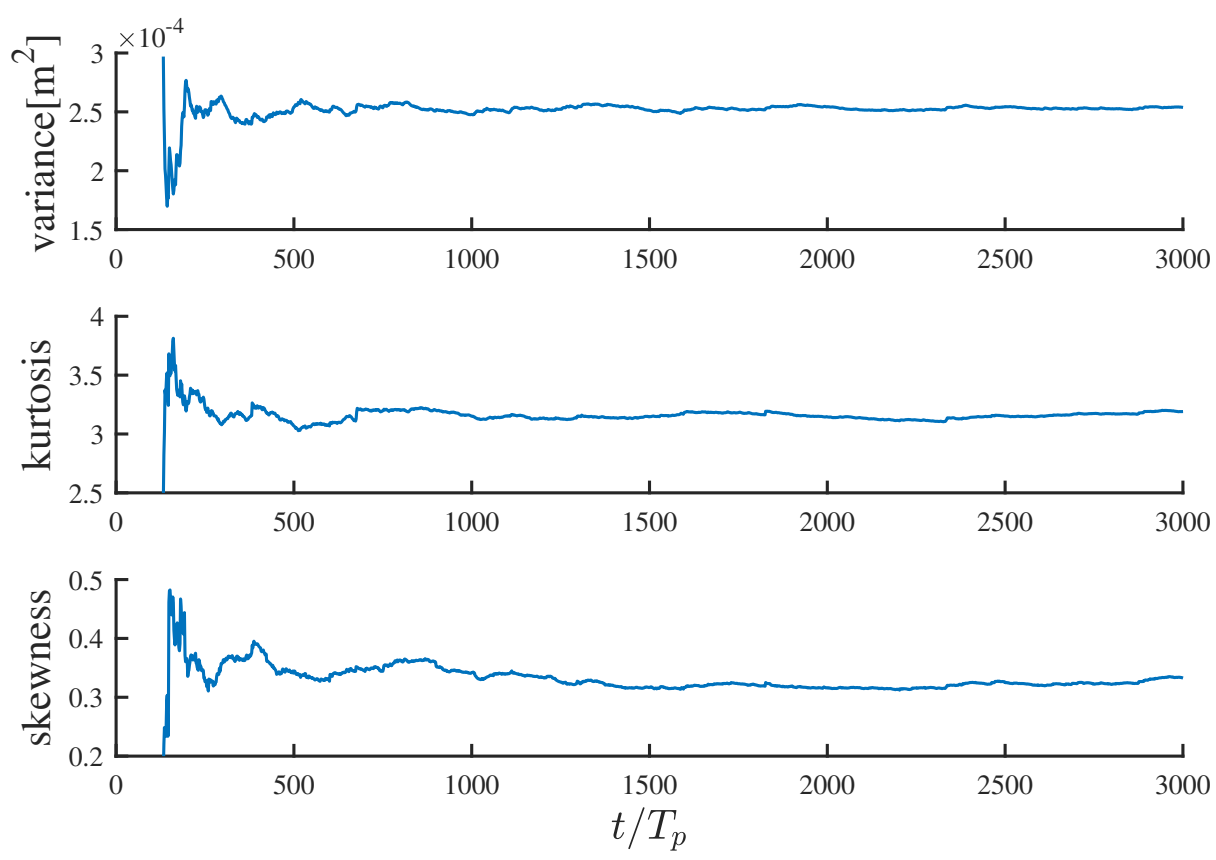

Figure 19: Statistical moments as a function of the length of time over which they are computed normalized by the peak period $T_{p}$.
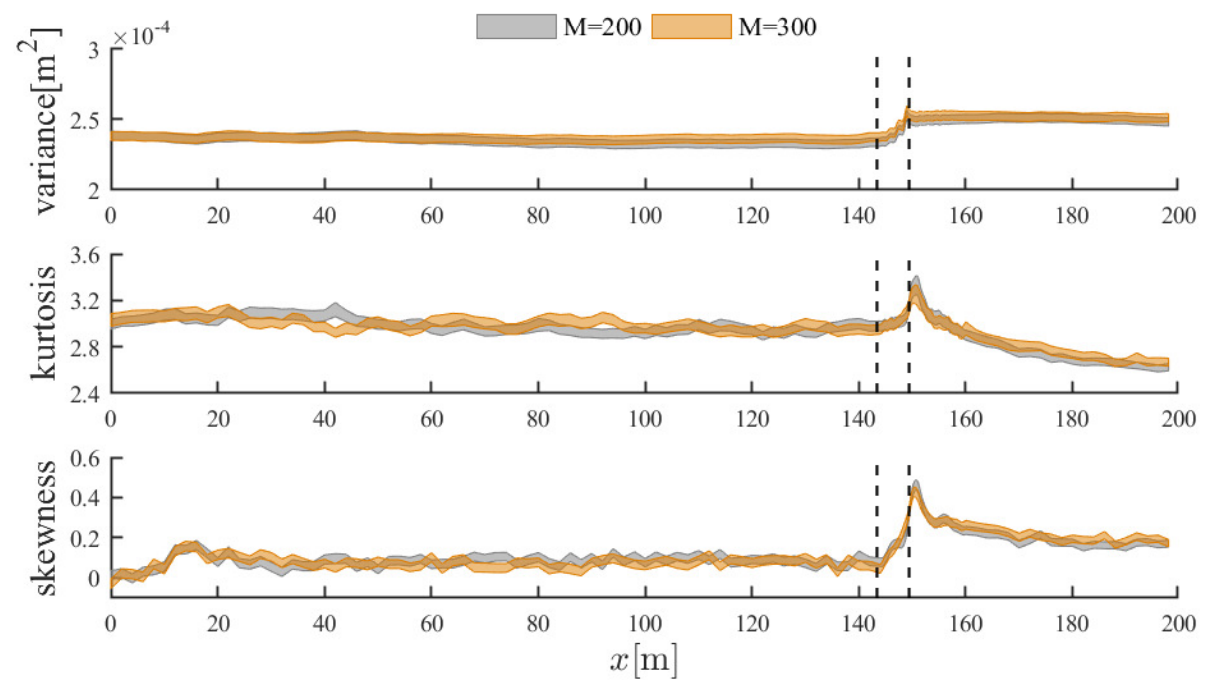

Figure 20: The variance $\left(\sigma^{2}\right)$, kurtosis $\left(\lambda_{4}\right)$ and skewness $\left(\lambda_{3}\right)$ for case A2 for different numbers or random wave components $M$. The shaded strips correspond to the $95 \%$ confidence intervals obtained from our numerical simulations. The dashed vertical lines denote the beginning and the end of the slope. 
[4] C. Tyler Bolles, K. Speer, and M. N. J. Moore. Anomalous wave statistics induced by abrupt depth change. Phys. Rev. Fluids, 4(1), 2019.

[5] C. A. Brebbia. The boundary element method for engineers. Pentech Press, 1978.

[6] T. Bunnik. Benchmark workshop on numerical wave modeling - description of test cases. Technical Report 70022-1-RD (MARIN, The Netherlands), 2010.

[7] G. Ducrozet and M. Gouin. Influence of varying bathymetry in rogue wave occurrence within undirectional and directional sea-states. J. Ocean Eng. Mar. Energy, 3:309-324, 2017.

[8] K. B. Dysthe, H. E. Krogstad, and P. Muller. Oceanic rogue waves. Annual Review of Fluid Mechanics, 40:287-310, 2008.

[9] F. Fedele, J. Brennan, S. P. De León, J. Dudley, and F. Dias. Real world ocean rogue waves explained without the modulational instability. Scientific Reports, 6:27715, 2016.

[10] C. J. Fitzgerald, P. H. Taylor, R. Eatock Taylor, J. Grice, and J. Zang. Phase manipulation and the harmonic components of ringing forces on a surface-piercing column. Proc. R. Soc. A., 470(20130847), 2014.

[11] J. D. Flanagan, F. Dias, E. Terray, B. Strong, and J. Dudley. Extreme water waves off the west coast of Ireland: Analysis of ADCP measurements. In The 26th International Ocean and Polar Engineering Conference, pages ISOPE-I-16-589, 2016.

[12] Y. Goda. Statistical variability of sea state parameters as a function of wave spectrum. Coastal Engineering in Japan, 31:39-52, 1988.

[13] O. Gramstad, H. Zeng, K. Trulsen, and G.K. Pedersen. Freak waves in weakly nonlinear unidirectional wave trains over a sloping bottom in shallow water. Physics of Fluids, 25:122103, 2013.

[14] K. Hirayama H. Kashima and N. Mori. Estimation of freak wave occurrence from deep to shallow water regions. Coast. Eng. Proc., 1:34-36, 2014.

[15] S. Haver. A possible freak wave event measured at the Draupner jacket January 1 1995. In Rogue Waves Workshop, pages 1-8, Brest, France, 2004.

[16] P. A. E. M. Janssen. Nonlinear four-wave interactions and freak waves. J. Phys. Oceanogr., 33:863-884, 2003.

[17] T.T. Janssen and T.H. Herbers. Nonlinear wave statistics in a focal zone. J. Phys. Oceanogr., 39:1948-1964, 2009.

[18] C. Kharif and E. Pelinovsky. Physical mechanisms of the rogue wave phenomenon. Eur. J. Mech. B-Fluid., 22:603-634, 2003.

[19] W. Koo and M. H. Kim. Freely floating-body simulation by a 2D fully nonlinear numerical wave tank. Ocean Engineering, 31(16):2011-2046, 2004.

[20] Y. J. Liu and N. Nishimura. The fast multipole boundary element method for potential problems: a tutorial. Engineering Analysis with Boudary Elements, 30:371-381, 2006.

[21] M. S. Longuet-Higgins. The statistical distribution of the heights of sea waves. J. Mar. Res., 11:1245, 1952.

[22] M. S. Longuet-Higgins and E. D. Cokelet. The deformation of steep surface waves on water. I. A numerical method of computation. Proc. R. Soc. Lond. A., 350:1-26, 1976.

[23] A. K. Magnusson and M. A. Donelan. The Andrea wave characteristics of a measured North Sea rogue wave. Journal of Offshore Mechanics and Arctic Engineering, 135:031108, 2013.

[24] A. J. Majda, M. N. J. Moore, and Di Qi. Statistical dynamical model to predict extreme events and anomalous features in shallow water waves with abrupt depth change. P. Natl. Acad. Sci. USA, 116(10):3982-3987, 2019. 
[25] M. L. McAllister, T. A. A. Adcock, P. H. Taylor, and T. S. van den Bremer. The set-down and set-up of directionally spread and crossing surface gravity wave groups. J Fluid Mech., 835:131-169, 2018.

[26] N. Nishimura. Fast multipole accelerated boundary integral equation methods. Applied Mechanics Reviews, 55:299-324, 2002.

[27] M. Onorato, S. Residori, U. Bortolozzo, A. Montina, and F. T. Arecchi. Rogue waves and their generating mechanisms in different physical contexts. Physics Reports, 528:47-89, 2013.

[28] M. Onorato, T. Waseda, A. Toffoli, L. Cavaleri, O. Gramstad, P. A. E. M. Janssen, T. Kinoshita, J. Monbaliu, N. Mori, A. R. Osborne, M. Serio, C. T. Stansberg, H. Tamura, and K. Trulsen. Statistical properties of directional ocean waves: the role of the modulational instability in the formation of extreme events. Physical Review Letters, 102:114502, 2009.

[29] E. Pan. Green's functions for geophysics: a review. Reports on Progress in Physics, 82(10):106801, 2019.

[30] A. Sergeeva, E. Pelinovsky, and T. Talipova. Nonlinear random wave fields in shallow water: Variable Korteweg-de Vries framework. Nat. Hazards Earth Syst. Sci., 11:110-118, 2011.

[31] K. Trulsen, A. Raustøl, S. Jorde, and L. Bæverfjord Rye. Extreme wave statistics of longcrested irregular waves over a shoal. J. Fluid Mech., 882(R2), 2020.

[32] K. Trulsen, H. Zeng, and O. Gramstad. Laboratory evidence of freak waves provoked by non-uniform bathymetry. Physics of Fluids, 24:097101, 2012.

[33] C. Viotti and F. Dias. Extreme waves induced by strong depth transitions: Fully nonlinear results. Physics of Fluids, 26:051705, 2014.

[34] T. Waseda, T. Kinoshita, and H. Tamura. Interplay of resonant and quasi-resonant interaction of the directional ocean waves. J. Phys. Oceanogr., 39:2351-2362, 2009.

[35] K. I. Yoshida. Applications of fast multipole method to boundary integral equation method. Kyoto: Kyoto University, 2001.

[36] H. Zeng and K. Trulsen. Evolution of skewness and kurtosis over a sloping bottom. Nat. Hazards Earth Syst. Sci., 12:631-638, 2012.

[37] J. Zhang, M. Benoit, O. Kimmoun, A. Chabchoub, and H. Hsu. Statistics of extreme waves in coastal waters: Large scale experiments and advanced numerical simulations. Fluids, 4:99, 2019. 